\title{
1 Niche-inspired synthetic matrices for epithelial organoid culture
}

2 Victor Hernandez-Gordillo ${ }^{1}$, Timothy Kassis ${ }^{1}$, Arinola Lampejo ${ }^{1}$, GiHun Choi ${ }^{1}$, Mario E.

3 Gamboa $^{1}$, Juan S. Gnecco ${ }^{1}$, David T. Breault ${ }^{2}$, Rebecca Carrier ${ }^{3}$, and Linda G. Griffith ${ }^{1}$

$4 \quad{ }^{1}$ Center for Gynepathology Research and Biological Engineering Department, Massachusetts Institute of Technology, 77

5 Massachusetts AV Cambridge MA, USA 02138. 2 Deparment of Pediatrics, Harvard Medical School, 300 Longwood Avenue,

6 Boston, MA 02115. ${ }^{3}$ Department of Chemical Engineering, Northeastern University, 208 Lake Hall, Boston, MA, USA ,02115.

8 Abstract

9 Epithelial organoids are now an important tool in fields ranging from regenerative medicine to

10 drug discovery. Organoid culture requires Matrigel, a complex, tumor-derived, extracellular

11 matrix. An alternative completely synthetic matrix could improve culture reproducibility, clarify

12 mechanistic phenomena, and enable applications involving human implantation. Here, we

13 designed synthetic matrices with tunable biomolecular and biophysical properties that allowed us

14 to identify critical gel parameters in organoid formation. Inspired by known epithelial integrin

15 expression in the proliferative niche of the human intestine, we identified an $\alpha 2 \beta 1$ integrin-binding

16 peptide as a critical component of the synthetic matrix that supports human duodenal colon and

17 endometrial organoid propagation. We show that organoids emerge from single cells, retain their

18 proliferative capacity, are functionally responsive to basolateral stimulation and have correct

19 apicobasal polarity upon induction of differentiation. The local biophysical presentation of the

20 cues, rather than bulk mechanical properties, appears to be the dominant parameter governing

21 epithelial cell proliferation and organoid formation in the synthetic matrix.

Introduction

24 Epithelial organoids are three-dimensional (3D) structures with microarchitecture, cellular composition and functions, similar to their native tissues ${ }^{1}$. They are used to model tissue homeostasis and pathophysiological processes and as in vitro models for drug development ${ }^{1-3}$.

27 However, their full potential has been hindered by the requirement for propagation in Matrigel, a 28 complex, tumor-derived extracellular matrix (ECM) containing a plethora of growth factors (GFs) 29 that are variable from lot-to-lot and exert unknown effects on organoid phenotype ${ }^{4-7}$. Envisioned 30 applications involving re-implantation of patient-derived organoids require Good Manufacturing 31 Practices (GMP) that exclude tumor-derived material ${ }^{8,9}$. Hence, there are many forces driving the 32 development of synthetic matrices in which all exogenous biological cues are known and defined. 
Towards this goal, Gjorevski et al., developed a synthetic poly(ethylene) glycol (PEG) gel modified with a fibronectin (FN)-derived RGD peptide or laminin (LMN)-derived peptides that supported mouse intestinal organoid growth but not differentiation. Differentiation was achieved in a semisynthetic matrix made with PEG and full-length $\mathrm{LMN}^{10}$. Human organoids survived in the PEG-RGD gel, although expansion, differentiation, and other features were not reported. Mouse organoid growth in the PEG gel appear to depend on the gel's bulk mechanical properties: stiff $(\sim 1.3 \mathrm{kPa})$ for organoid formation and soft $(\sim 300 \mathrm{~Pa})$ for differentiation ${ }^{10}$. The gel's bulk mechanical properties were modulated by processes that simultaneously also affect other gel properties, such as ligand accessibility, clustering, and nanomechanical responses to cell-generated forces after ligand binding ${ }^{11-19}$. The observation that organoids grow significantly better in

11 Matrigel or in hydrogels made with low concentrations of purified LMN than in PEG-RGD or 12 collagen gels ${ }^{10,20-23}$ suggests that specific cell-matrix affinity interactions might play a critical role 13 in organoid formation and differentiation. Untangling the relative contributions of bulk mechanical 14 properties from other gel biophysical and biomolecular properties on cell proliferation and organoid formation is challenging.

16 Inspired by integrins expression in the native tissue, here we create synthetic matrices with 17 tunable biophysical and biomolecular properties that support organoid formation and differentiation across multiple donors and tissue types. The local biophysical presentation and the identity of the integrin-binding motif, rather than bulk biomechanical properties or matrix

20 degradation, appear to be the dominant variables governing epithelial cell proliferation and 21 organoid formation in the synthetic matrix.

\section{Niche-inspired synthetic ECM}

24 Intestinal stem cells (ISCs) at the bottom of the crypt express $\alpha 2 \beta 1$ and $\alpha 5 \beta 1$ integrins ${ }^{24-29}$. 25 Consequently, ligands for these receptors are attractive components of synthetic ECM. We created 26 a modular synthetic ECM by combining 8-arm vinyl sulfone-activated PEG macromers partially 27 modified with integrin-binding peptides and matrix-binding peptides together with peptide 28 crosslinkers containing a matrix-metalloproteinase (MMP)-sensitive degradation site. In our 29 design, we focused on three variables that influence integrin-ligand interactions: a) identity of the 30 integrin-binding ligand b) biophysical properties of ligand presentation and c) matrix

31 biomechanical properties at local and macroscale (Fig 1a). We also included cell-mediated matrix 
1 remodeling (via proteases and matrix deposition) and dynamic softening, via Sortase degradation, as additional variables.

As PEG chains are highly flexible in aqueous solution, they can be tailored in length to modulate ligand accessibility ${ }^{1-19}$ and in combination with crosslinking density, also modulate local and bulk mechanical properties ${ }^{30-32}$. In our design, the arm PEG lengths were modulated to either $\sim 20 \mathrm{~nm}$ or $\sim 40 \mathrm{~nm}$ (when fully extended from the central polymer core) by using 8-arm PEG macromers of $20 \mathrm{kDa}$ or $40 \mathrm{kDa}$, respectively (Fig 1a). To parse the role of the $\alpha 2 \beta 1$ and $\alpha 5 \beta 1$ integrins on cell survival and organoid formation, we created matrices using single, or mixed (1:1, $\mathrm{W} / \mathrm{v}$ ) macromers with a fixed crosslinking density. To engage the $\alpha 5 \beta 1$ integrin, we functionalized the PEG macromers with an FN-derived peptide that contains the $\boldsymbol{R} \boldsymbol{G D}$ motif and the PHSRN

11 synergy site (PEG-PHSRN-K-RGD)$)^{11,33,34}$. Likewise, to engage the $\alpha 2 \beta 1$ integrin, we used a 12 collagen I-derived peptide harboring the $\boldsymbol{G F O G E R}$ sequence (PEG-GFOGER$)^{35}$.

13 We also considered that initial integrin engagement is only a first step, with possible subsequent 14 interactions mediated by matrix that cells produce and assemble locally ${ }^{36,37}$. Hence, we included 15 peptides with affinity for FN, collagen IV (C-IV), and LMN (FN-binder and BM-binder, respectively) ${ }^{38-41}$. Lastly, we considered that organoid growth might require matrix degradation,

17 thus we used a protease-sensitive crosslinker concatenated with a site susceptible to cleavage with 18 exogenously-added transpeptidase SrtA enzyme to enable cell-mediated matrix remodeling and 19 recovery of intact organoids, respectively ${ }^{38}$.

20 Six synthetic matrix formulations (40kDa-PHSRN-K-RGD, 40kDa-GFOGER, 20kDa21 PHSRN-K-RGD，20kDa-GFOGER，40/20kDa-PHSRN-K-RGD，40/20kDa-GFOGER) that

22 varied in bulk mechanical properties but contained similar crosslinker density and equal nominal 23 concentration of integrin-, BM- and FN-binding peptides, were made. In the PEG-PHSRN-K-

$24 \boldsymbol{R G D}$ matrices, the RGD and PHSRN sites are presented in a branched configuration to mimic the 25 biophysical presentation found in native $\mathrm{FN}^{11,42}$ whereas in the PEG-GFOGER , three cell-binding cues are presented as a triple helix, thus mimicking the biophysical presentation of collagen fibers $27 \quad(\text { Fig } 1 \mathrm{a})^{35}$. 
1 Donor-to-donor and species variability is known to affect organoid growth ${ }^{43,44}$. Thus, to test the 2 synthetic matrices, we selected seven human donors (six duodenal and one colon) that varied in sex, age and pathological state, and one mouse colon donor. Dissociated single cells were embedded in the synthetic matrices and then cultured in expansion medium (EM). To facilitate the identification of a suitable matrix for organoid culture, we used the organoid diameter as a proxy for cell proliferation and organoid growth (Extended Data Fig 1$)^{45}$.

Overall, organoids emerging in both Matrigel and in the synthetic matrices had a wide range of size distributions, but all were spherical with a single layer of thin epithelial cells. We considered this phenotype as undifferentiated and potentially stem-enriched organoids (Fig 1b, Extended Data Fig 2a). We designated these spherical organoids as "enteroids" and reserved the term "organoid"

11 for enteroids that underwent differentiation and adopted a thick columnar epithelial cell layer.

The synthetic matrices supported enteroid formation from human and mouse donors with 13 various degree of success (Fig 1b,d, Extended Data Fig 2). Compared to Matrigel, human enteroids 14 in the synthetic matrices were smaller. Interestingly, human colon enteroids grew slower, in 15 Matrigel and the synthetic matrices, compared to the duodenal enteroids. In contrast, mouse colon 16 enteroids grew faster than human colon enteroids in Matrigel or the synthetic matrices. In some 17 matrix conditions mouse colon enteroids reached sizes similar to mouse colon enteroids in 18 Matrigel, ( Extended Data Fig 2b).

19 From the two sets of synthetic matrices, the PEG-GFOGER ( $\alpha 2 \beta 1)$ series supported enteroid 20 formation, across all donors, to a greater extent than the PEG-PHSR $\boldsymbol{N}-\boldsymbol{K}-\boldsymbol{R} \boldsymbol{G D}(\alpha 5 \beta 1)$ series, even 21 though the encapsulated cells expressed $\alpha 2, \alpha 5$, and $\beta 1$ integrins (Extended Data Fig 3a). The 22 ability of the PEG-GFOGER series to support enteroid formation is not due to bulk mechanical differences, as the 20kDa-PHSRN-K-RGD and the 20kDa-GFOGER matrices have similar mechanical properties, yet we observed significant differences in enteroid diameters (Fig 1c-d, 25 Extended Data Fig 2). We also noted that the GFOGER bioactivity is context dependent, as matrices with relatively high swelling, lower storage modulus, and longer PEG arms, failed to 27 promote robust enteroid formation (Compare 40kDa-GFOGER vs 20kDa-GFOGER). Matrices 28 made with the 1:1 (w/v) mixed macromers (40/20kDa-GFOGER) rescued the GFOGER 29 bioactivity. This is more likely because $\sim 70 \%$ of PEG arms in the $40 / 20 \mathrm{kDa}-\boldsymbol{G F O G E R}$ matrix 30 correspond to the 20kDa-PEG macromer, which results in a matrix of similar biomechanical 31 properties as the 20kDa-GFOGER (Fig 1b-d). This phenomenon of context-dependent success of 
1 enteroid formation was not observed in the PEG-PHSR $\boldsymbol{N}-\boldsymbol{K}-\boldsymbol{R} \boldsymbol{G D}$ series, in part because enteroid emergence was relatively poor.

Collectively, the data shows that intestinal cell proliferation and enteroid formation occurs in a narrow set of matrix mechanical properties in human enteroids and in a broader range in mouse enteroids. Notably, our results highlight the significance of using a variety of donors and tissue types, as matrices that supported mouse enteroids failed with human donors. Across the human donors, we also noticed donor-to-donor variability on enteroid diameter and size distribution in the synthetic matrices that mirrored the donor-to-donor variability observed in Matrigel. Compared to Matrigel, the synthetic ECM supported enteroid formation efficiency of $47 \%$ (duodenal) and $40 \%$ (colon) (Fig 1e). These differences could be due to the number of residual growth factors and

11 cytokines in Matrigel that are absent in our synthetic ECM.

\section{Enteroid growth depends on GFOGER/ $\alpha 2 \beta 1$}

14 Modulation of the ligand's properties in the 20kDa-GFOGER matrix revealed that enteroid 15 formation depends on the $\alpha 2 \beta 1$ integrin binding to the $\boldsymbol{G F O G E R}$ peptide (Fig 2a) in a dose16 dependent manner (Fig 2b-c). An inactive form of the $\boldsymbol{G F O G E R}$ peptide $(\boldsymbol{G F O} \boldsymbol{G} \underline{\boldsymbol{D}} \boldsymbol{R})^{35}$ caused an 17 increase in the number of dead cells and failed to promote enteroid formation (Fig $2 \mathrm{a}$ and $\mathrm{c}$ ). Thus, 18 engaging $\alpha 2 \beta 1$ is not only essential for enteroid formation but also needed to maintain a population of cells that do not form enteroids, yet remain viable.

Changes in the identity and concentration of the crosslinker also affected enteroid formation. Enteroids emerged from single cells encapsulated in a non-degradable synthetic ECM, but by 6 days were smaller and less abundant compared to enteroids in matrices made with a celldegradable crosslinker (Extended Data Fig 3b). This suggests that the matrix's microarchitecture can support initial cell proliferation to establish enteroids, but later stages in growth might require simultaneous matrix degradation. Crosslinker densities of 45 and $50 \%$ at $1.5 \mathrm{mM}$ nominal concentration of $\boldsymbol{G F O G E R}$ appears the most favorable environment for enteroid growth. Lower crosslinker densities caused greater hydrogel swelling and reduced enteroid growth, possible due

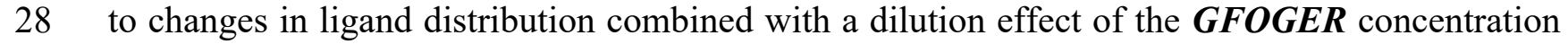
within the matrix (Extended Data Fig 3c).

30 Because ligand presentation and clustering affect integrin binding, we wondered if the higher

31 GFOGER bioactivity compared to PHSRN-K-RGDS was due to the triple-helical nature of the 
1 peptide that extends and clusters the ligand away from the PEG polymer core compared to the

2 branched and shorter configuration of the PHSRN-K-RGDS. Longer and clustered integrin

3 binding peptides have been shown to increase accessibility and integrin binding which results in

4 higher cell proliferation ${ }^{46-48}$. Thus, we hypothesized that increasing the $\alpha 5 \beta 1$ ligand accessibility

5 and clustering, while maintaining the bulk mechanical properties constant, would result in

6 increased enteroid formation. We synthesized three peptides harboring the $\alpha 5 \beta 1$ ligand (Arg-Gly-

7 Asp,Ser, RGDS); a short linear peptide (RGDS), a longer extended ligand (G11RGDS) and a

8 longer and clustered RGDS ligand (CMPRGDS $)^{47}$ (Fig 2d-e). Although we saw an increase in

9 enteroid formation efficiency and diameters with the extended (G11RGDS) and clustered

10 (CMPRGDS) peptides compared to linear $\boldsymbol{R} \boldsymbol{G D S}$, they were significantly smaller were less

11 efficient in enteroid formation than the GFOGER matrix (Fig 2f-g).

12 Collectively, the previous data point to a mechanism for enteroid formation that appears to be 13 dependent on the GFOGER- $\alpha 2 \beta 1$ integrin interaction, as extending and clustering the a5 $\beta 1$

14 (RGDS) ligand failed to promote robust enteroid growth. Further, GFOGER presentation appears

15 to be favored by shorter $(\sim 20 \mathrm{~nm})$ compared to longer $(\sim 40 \mathrm{~nm})$ PEG arms, possibly due to a

16 favorable nanomechanical response when the cells pull on the ligand ${ }^{11,13,17,19}$.

18 Enteroid characterization in the synthetic ECM

19 To gain more insight into the growth of human enteroids in the synthetic ECM, we followed the

20 formation of enteroids from single cells (Fig 3a, Extended Data Fig 4a). Time-lapse live imaging

21 from days 1 to 2 and 2 to 3 after encapsulation revealed faster growth in Matrigel compared to the

22 synthetic ECM (Supplementary videos). A clear lumen was visible by day three in Matrigel and

23 by day four in the synthetic ECM (Fig 3a). By day six, cells that failed to form enteroids were still

24 viable (Fig 3b). Viable single cells that did not form enteroids were observed in various human

25 donors (Extended Data Fig 4b). Time-lapse live cell imaging from day 4 to 6 revealed that

26 enteroids in Matrigel and the synthetic ECM grew in a manner reminiscent of fluid pressure

27 oscillations ${ }^{49,50}$. Time-lapse live cell imaging also captured epithelial cells being extruded into the

28 lumen in Matrigel and the synthetic ECM, suggesting the establishment of a functional and

29 polarized epithelial layer (see red arrows in Fig 3d and supplementary videos). Actin staining

30 further confirmed correct enteroid polarization across multiple donors (Extended Data Fig 4c). 
As enteroids in the synthetic ECM, on average, had a delayed growth, we extended our organoid diameter analysis for up to ten days. Although enteroids in the synthetic ECM continued to grow (Fig 3b-c), the size distribution of the overall population at day 10 did not reach that of the population in Matrigel at day 6. Interestingly, enteroids in Matrigel at days 8 and 10 started to fuse and created a mass of interconnected cells at the bottom of the well accompanied by significant cell death, possibly due to Matrigel degradation and dissolution. We did not observe such a phenomenon in the synthetic ECM, which is an important feature for long-term culture and emerging application of organoids.

Properly polarized and functional organoids can be used to study adaptive epithelial responses $^{51}$. To test if enteroids in the synthetic ECM were functionally responsive to basolateral 11 stimulation, we treated six-day old enteroids with prostaglandin E2 (PGE2) and forskolin (FKL).

12 PGE2 and FKL have been shown to induce rapid morphological changes characterized by an 13 increase in organoid diameter ${ }^{51,52}$. Upon PGE2 and FKL treatment, enteroids in the synthetic ECM 14 increased in diameter, similar to the response we observed with enteroids in Matrigel (Extended 15 Data Fig 6d-e, Supplementary videos). This response suggests that the synthetic ECM not only 16 supports functionally responsive enteroids but is also flexible enough to accommodate a rapid 17 increase in enteroid size that is more likely independent of matrix degradation.

\section{Enteroids in the synthetic ECM retain their proliferative capacity}

20 Epithelial organoids can be cultured continuously due to an undifferentiated stem cell population 21 that, when re-embedded in Matrigel, gives rise to new organoids ${ }^{53}$. To determine if a population 22 of undifferentiated and proliferative cells existed in enteroids emerging in the synthetic ECM, we 23 performed quantitative PCR (qPCR) for five genes associated with active stem cells, six genes 24 associated with quiescent stem cells ( +4 position) and nine genes associated with progenitor cells 2554,55 . Overall, in the two duodenal donors tested, we observed similar levels of gene expression of 26 active and quiescent stem and progenitor genes in enteroids emerging in the synthetic ECM 27 compared to Matrigel, except for SMOC2, MMP7, and ATOH1 genes that showed more 28 variability (Fig 4a and Extended Data Fig 5a).

29 As enteroids in the synthetic matrix appear to retain a similar gene expression profile to 30 enteroids in Matrigel, we sought to investigate if they contained a proliferative cell population 31 capable of forming new enteroids when re-embedded in new synthetic ECM or Matrigel. Six-day 
1 old enteroids were collected from the synthetic ECM and then processed (see Methods) to generate 2 single cells to re-embed into new synthetic ECM or Matrigel (Extended Data Fig 6a). We 3 performed this procedure for three consecutive passages with two duodenal donors. Quantification

4 of the total number of cells recovered after each passage in the synthetic ECM revealed a 1.5 to 2-

5 fold increase in cell expansion relative to the initial number of encapsulated cells (Extended Data

6 Fig 6b). As described before, we also noted a population of cells that failed to form enteroids but

7 remained viable. This cell population was also included in the calculation of fold increase in

8 Extended Data Fig 6b. Interestingly, when single cells from organoids grown in the synthetic ECM were embedded in Matrigel there was more than 14-fold increase in cell expansion as the majority of single cells formed enteroids. This might be due to the number of residual growth factors present

11 in Matrigel that could induce a proliferative phenotype in otherwise quiescent cells ${ }^{55,56}$. This

12 observation furthers highlights the significance of developing a suitable matrix to uncover

13 biological processes in epithelial organoids masked when using ill-defined Matrigel hydrogels.

14 Quantification of enteroid diameters after each passage further revealed a wide range of sizes in 15 the population, but similar to previous results, enteroids in Matrigel were, on average, bigger than

16 those in the synthetic ECM (Fig 4b, Extended Data Fig 5b, 6c). The wide range in enteroid 17 diameter suggests that enteroids recovered from the synthetic ECM, similar to those recovered from Matrigel, contain a population of cells that vary in their proliferative capacity.

To further characterize the duodenal enteroids in the synthetic ECM, we labeled them with 5ethynyl-2'-deoxyuridine (EdU) to identify proliferative cells, then performed immunostaining to identify Paneth (Lyz) cells, as Paneth cells are part of the intestinal stem cell niche in vivo. We observed a number of EdU and lyz positive cells in enteroids grown in Matrigel and synthetic ECM in two donors tested. Further, enteroids also showed E-cadherin and villin, typical markers

24 for intestinal epithelial cells Collagen IV and CD44-v6 (a hyaluronic acid receptor expressed at 25 the bottom of the crypt) ${ }^{57}$ were also detected in enteroids growing in the synthetic ECM (Fig 4c$26 \mathrm{~d}$ and Extended Data Fig 5c).

\section{Enteroid differentiation in synthetic ECM}

29 Stem-enriched enteroids in Matrigel undergo differentiation when switched to differentiation 30 medium (DM) lacking Wnt3a, a ligand for the Frizzled receptor that enhances the activity of the 31 LGR5 ligand, R-spondin1, in LGR5-positive ISCs ${ }^{53}$, or DM made with lower concentrations of L- 
1 WRN (Wnt3a, R-spondin1 and Noggin) conditioned medium (L-WRN-DM) ${ }^{63}$. Stem-enriched

2 mouse enteroids grown in a synthetic ECM, in contrast, undergo rapid cell death upon switching 3 to DM, due to a rapid loss of resident stem cells ${ }^{10}$. We hypothesized that to allow differentiation

4 in a synthetic ECM we would need to preserve a pool of stem cells while allowing the emergence

5 of differentiated cells. Thus, enteroids were differentiated using a lower concentration of L-WRN

6 conditioned medium. Human duodenal and colon enteroids were grown for six days in EM (50\%

7 L-WRN) then switched to L-WRN-DM (25\% L-WRN) or DM. Similar to previous results with 8 mouse organoid differentiation in a synthetic ECM, removal of Wnt3a (DM) caused rapid cell 9 death of human enteroids that was not observed in enteroids differentiated in Matrigel (Data not 10 shown). In the presence of L-WRN-DM, human enteroids adopted diverse phenotypes with some 11 enteroids showing typical hallmarks of differentiation (thick columnar cells and accumulation of 12 apoptotic cells in the lumen), and in some instances adopting folded 3D structures (Fig 5a). Mouse 13 intestinal enteroids in the synthetic ECM also showed hallmarks of differentiation (Extended Data 14 Fig 9a-b). Immunostaining analysis of human and mouse organoids also revealed correct 15 polarization as shown with a diverse array of apical (F-actin, NHE3, DPP4, villin) and basolateral 16 markers (collagen IV, LMN, CD44v6). Proliferative cells (Ki67 or EdU), Paneth cells (Lyz), and 17 Goblet cells (Muc2) were also observed in the differentiated duodenal and colon enteroids (Fig 18 5b-c, Extended Data Figure 7-8, 9c). In summary, the data shows that enteroids emerging in the 19 synthetic ECM are composed of stem cells that can undergo differentiation to a similar extent to 20 enteroids emerging in Matrigel.

\section{Endometrial organoids in synthetic ECM}

23 We extended our study to include human endometrial organoids, as similar to intestinal organoids, 24 they are composed of a single layer of epithelial cells surrounding a lumen. Interestingly, 25 endometrial glands in vivo express the $\alpha 2 \beta 1$ and $\alpha \mathrm{V} \beta 1$ integrin receptors ${ }^{59-61}$. Thus, we selected 26 a panel of synthetic matrices to engage the $\alpha 2 \beta 1(20 \mathrm{kDa}-\boldsymbol{G F O G E R})$ or the $\alpha \mathrm{V} \beta 1(20 \mathrm{kDa}-$ 27 PHSRN-K-RGD, 20kDa-RGDS, 20kDa-G11RGDS and 20kDa-CMPRGDS) integrins. Single 28 cells embedded in the synthetic matrices formed globular organoids with thick columnar cells 29 surrounding an empty lumen. Similar to intestinal enteroids, endometrial organoids in Matrigel 30 were bigger and more abundant than organoids in the synthetic matrices. Nonetheless, from all the 31 synthetic matrices tested, the 20kDa-GFOGER ( $\alpha 2 \beta 1)$ supported bigger and more abundant 
1 endometrial organoids (Fig 6a-b). Endometrial organoids, in the synthetic ECM, showed

2 proliferative cells (EdU), epithelial (EpCAM) and apicobasal markers (actin, LMN) similar to

3 those grown in Matrigel (Fig 6c-d).

\section{Conclusions}

6 Designing a synthetic matrix that supports robust organoid formation and differentiation from a

7 spectrum of different donor origins has been challenging due to differences between mouse and

8 human organoids along with donor-to-donor variability. We report for the first time, to our

9 knowledge, a fully synthetic matrix that support organoid formation from different tissue types

10 and epithelial organs. Inspired by integrin expression in the native tissue, we found that the local

11 biophysical presentation of an $\alpha 2 \beta 1$ integrin-binding peptide, rather than the bulk gel's mechanical

12 properties, appears to be the dominant variable governing epithelial cell proliferation and organoid

13 formation. We found that enteroids emerging in the synthetic ECM are, on average, less numerous

14 and showed delayed growth compared to Matrigel. This discrepancy could be attributed to:

15 residual GFs in Matrigel that affect organoid biology in unknown ways; additional matrix

16 components (e.g., laminins) that stimulate additional signaling pathways; the propensity of

17 Matrigel to bind GFs present in the culture media and serve as a depot for later release; or any

18 combination of these. Interestingly, single cells that do not progress to enteroids remain viable in

19 the synthetic ECM for over a week. Thus, the synthetic matrix may allow interrogation and

20 discovery of factors that impact organoid biology.

21 Although not fully explored here, the synthetic ECM offers additional benefits such as on-

22 demand dissolution to recover intact cells and cell-secreted metabolites ${ }^{38}$ and incorporation of

23 small peptide that sequester cell-secreted matrix for an in-depth investigation of cell-matrix

24 deposition in tissue formation ${ }^{39}$. We expect that these features will be of great utility to uncover

25 complex and dynamic cell-cell communications in emerging stromal-epithelial co-culture

26 systems $^{39}$. We further envision that the synthetic matrix can also be used in microfabrication

27 approaches aimed to replicate the intestinal topography ${ }^{62,63}$. Such systems would also require cues

28 to engage $\alpha 2 \beta 1$-expressing cells to preserve a proliferative compartment. In conclusion, the matrix

29 developed here has the potential to be a "one size fits all" to multiple applications. To facilitate its

30 adoption by the organoid and bioengineering community, we used commercially available and

31 made-to-order reagents that require minimal manipulation and common laboratory equipment. 
1. Drost, J. \& Clevers, H. Organoids in cancer research. Nature Reviews Cancer 18, 407-418 (2018).

2. $\quad$ Rossi, G., Manfrin, A. \& Lutolf, M. P. Progress and potential in organoid research. Nature Reviews Genetics (2018). doi:10.1038/s41576-018-0051-9

3. Kaushik, G., Ponnusamy, M. P. \& Batra, S. K. Concise Review: Current Status of ThreeDimensional Organoids as Preclinical Models. Stem Cells 36, 1329-1340 (2018).

4. Sato, T. et al. Single Lgr5 stem cells build crypt-villus structures in vitro without a mesenchymal niche. Nature 459, 262-5 (2009).

5. Talbot, N. C. \& Caperna, T. J. Proteome array identification of bioactive soluble proteins/peptides in Matrigel: relevance to stem cell responses. Cytotechnology 67, 873883 (2015).

6. Hughes, C. S., Postovit, L. M. \& Lajoie, G. A. Matrigel: a complex protein mixture required for optimal growth of cell culture. Proteomics 10, 1886-1890 (2010).

7. Boretto, M. et al. Patient-derived organoids from endometrial disease capture clinical heterogeneity and are amenable to drug screening. Nat. Cell Biol. 21, 1041-1051 (2019).

8. Nakamura, T. \& Sato, T. Advancing Intestinal Organoid Technology Toward Regenerative Medicine. CMGH 5, 51-60 (2018).

9. Cruz-Acuña, R. et al. Synthetic hydrogels for human intestinal organoid generation and colonic wound repair. Nat. Cell Biol. 19, 1326-1335 (2017).

10. Gjorevski, N. et al. Designer matrices for intestinal stem cell and organoid culture. Nat. Publ. Gr. 539, 560-564 (2016).

11. Kuhlman, W., Taniguchi, I., Griffith, L. G. \& Mayes, A. M. Interplay between PEO tether length and ligand spacing governs cell spreading on RGD-modified PMMA-g-PEO comb copolymers. Biomacromolecules 8, 3206-3213 (2007).

12. Lopina, S. T., Wu, G., Merrill, E. W. \& Griffith-Cima, L. Hepatocyte culture on carbohydrate-modified star polyethylene oxide hydrogels. Biomaterials 17, 559-569 (1996).

13. Attwood, S. J. et al. Adhesive ligand tether length affects the size and length of focal adhesions and influences cell spreading and attachment. Sci. Rep. 6, (2016).

14. Wilson, M. J., Liliensiek, S. J., Murphy, C. J., Murphy, W. L. \& Nealey, P. F. Hydrogels with well-defined peptide-hydrogel spacing and concentration: Impact on epithelial cell behavior. Soft Matter 8, 390-398 (2012).

15. Rahil, Z. et al. Nanoscale mechanics guides cellular decision making. Integr. Biol. (United Kingdom) 8, 929-935 (2016).

16. Koo, L. Y., Irvine, D. J., Mayes, A. M., Lauffenburger, D. \& Griffith, L. G. Co-regulation of cell adhesion by nanoscale RGD organization and mechanical stimulus. J. Cell Sci. 115, 1423-1433 (2002).

17. Maheshwari, G., Brown, G., Lauffenburger, D. A., Wells, A. \& Griffith, L. G. Cell adhesion and motility depend on nanoscale RGD clustering. J. Cell Sci. 113 ( Pt 1, 16771686 (2000).

18. Houseman, B. T. \& Mrksich, M. The microenvironment of immobilized Arg-Gly-Asp peptides is an important determinant of cell adhesion. Biomaterials 22, 943-955 (2001).

19. Ebara, M. et al. The effect of extensible PEG tethers on shielding between grafted thermo- 
responsive polymer chains and integrin-RGD binding. Biomaterials 29, 3650-3655 (2008).

20. Broguiere, N. et al. Growth of Epithelial Organoids in a Defined Hydrogel. Adv. Mater. 30, 1801621 (2018).

21. Fujii, M. et al. Human Intestinal Organoids Maintain Self-Renewal Capacity and Cellular Diversity in Niche-Inspired Culture Condition. Cell Stem Cell 23, 787-793.e6 (2018).

22. Ahmad, A. A. et al. Optimization of 3-D organotypic primary colonic cultures for organon-chip applications. J Biol Eng 8, 9 (2014).

23. Soofi, S. S., Last, J. A., Liliensiek, S. J., Nealey, P. F. \& Murphy, C. J. The elastic modulus of Matrigel ${ }^{\mathrm{TM}}$ as determined by atomic force microscopy. J. Struct. Biol. 167, 216-219 (2009).

24. Muñoz, J. et al. The Lgr5 intestinal stem cell signature: Robust expression of proposed quiescent ' +4' cell markers. EMBO J. 31, 3079-3091 (2012).

25. Benoit, Y. D. et al. Integrin $\alpha 8 \beta 1$ confers anoikis susceptibility to human intestinal epithelial crypt cells. Biochem. Biophys. Res. Commun. 399, 434-439 (2010).

26. Benoit, Y. D., Groulx, J.-F., Gagné, D. \& Beaulieu, J.-F. RGD-Dependent Epithelial CellMatrix Interactions in the Human Intestinal Crypt. J. Signal Transduct. 2012, 1-10 (2012).

27. Starchenko, A. et al. Clustering of integrin $\alpha 5$ at the lateral membrane restores epithelial polarity in invasive colorectal cancer cells. Mol. Biol. Cell 28, 1288-1300 (2017).

28. Patey, N. et al. Distribution of cell adhesion molecules in infants with intestinal epithelial dysplasia (tufting enteropathy). Gastroenterology 113, 833-843 (1997).

29. Stallmach, A., Von Lampe, B., Matthes, H., Bornhoft, G. \& Riecken, E. O. Diminished expression of integrin adhesion molecules on human colonic epithelial cells during the benign to malign tumour transformation. Gut 33, 342-346 (1992).

30. Offeddu, G. S., Axpe, E., Harley, B. A. C. \& Oyen, M. L. Relationship between permeability and diffusivity in polyethylene glycol hydrogels. AIP $A d v .8,(2018)$.

31. Kloxin, A. M., Tibbitt, M. W., Kasko, A. M., Fairbairn, J. A. \& Anseth, K. S. Tunable hydrogels for external manipulation of cellular microenvironments through controlled photodegradation. Adv. Mater. 22, 61-66 (2010).

32. Kim, J. et al. Characterization of the crosslinking kinetics of multi-arm poly(ethylene glycol) hydrogels formed via Michael-type addition. Soft Matter 12, 2076-2085 (2016).

33. Feng, Y. \& Mrksich, M. The synergy peptide PHSRN and the adhesion peptide RGD mediate cell adhesion through a common mechanism. Biochemistry 43, 15811-15821 (2004).

34. Huettner, N., Dargaville, T. R. \& Forget, A. Discovering Cell-Adhesion Peptides in Tissue Engineering: Beyond RGD. Trends in Biotechnology 36, 372-383 (2018).

35. Knight, C. G. et al. The collagen-binding a-domains of integrins $\alpha 1 / \beta 1$ and $\alpha 2 / \beta 1$ recognize the same specific amino acid sequence, GFOGER, in native (triple- helical) collagens. J. Biol. Chem. 275, 35-40 (2000).

36. Hughes, C. et al. Mass Spectrometry-based Proteomic Analysis of the Matrix Microenvironment in Pluripotent Stem Cell Culture. Mol. Cell. Proteomics 11, 1924-1936 (2012).

37. Devaud, Y. R. et al. Label-Free Quantification Proteomics for the Identification of Mesenchymal Stromal Cell Matrisome Inside 3D Poly(Ethylene Glycol) Hydrogels. Adv. Healthc. Mater. 7, 1800534 (2018). 
38. Valdez, J. et al. On-demand dissolution of modular, synthetic extracellular matrix reveals local epithelial-stromal communication networks. Biomaterials 130, 90-103 (2017).

39. Cook, C. D. et al. Local remodeling of synthetic extracellular matrix microenvironments by co-cultured endometrial epithelial and stromal cells enables long-term dynamic physiological function. Integr. Biol. 9, 271-289 (2017).

40. Gao, X. \& Groves, M. J. Fibronectin-binding peptides. I. Isolation and characterization of two unique fibronectin-binding peptides from gelatin. Eur. J. Pharm. Biopharm. 45, 275284 (1998).

41. Johnson, G. \& Moore, S. W. Identification of a structural site on acetylcholinesterase that promotes neurite outgrowth and binds laminin-1 and collagen IV. Biochem. Biophys. Res. Commun. 319, 448-455 (2004).

42. Kao, W. J., Lee, D., Schense, J. C. \& Hubbell, J. A. Fibronectin modulates macrophage adhesion and FBGC formation: The role of RGD, PHSRN, and PRRARV domains. $J$. Biomed. Mater. Res. 55, 79-88 (2001).

43. Suzuki, K. et al. Single cell analysis of Crohn's disease patient-derived small intestinal organoids reveals disease activity-dependent modification of stem cell properties. $J$. Gastroenterol. 53, 1035-1047 (2018).

44. Kraiczy, J. et al. DNA methylation defines regional identity of human intestinal epithelial organoids and undergoes dynamic changes during development. Gut 68, 49-61 (2019).

45. Kassis, T., Hernandez-Gordillo, V., Langer, R. \& Griffith, L. G. OrgaQuant: Human Intestinal Organoid Localization and Quantification Using Deep Convolutional Neural Networks. Sci. Rep. 9, 12479 (2019).

46. Salinas, C. N. \& Anseth, K. S. The influence of the RGD peptide motif and its contextual presentation in PEG gels on human mesenchymal stem cell viability. J. Tissue Eng. Regen. Med. 2, 296-304 (2008).

47. Hernandez-Gordillo, V. \& Chmielewski, J. Mimicking the extracellular matrix with functionalized, metal-assembled collagen peptide scaffolds. Biomaterials 35, 7363-7373 (2014).

48. Petrie, T. A. et al. Tissue engineering: Multivalent integrin-specific ligands enhance tissue healing and biomaterial integration. Sci. Transl. Med. 2, 45ra60 (2010).

49. Ruiz-Herrero, T., Kévin Alessandri, K., Gurchenkov, B. V, Nassoy, P. \& Mahadevan, L. Organ size control via hydraulically gated oscillations. (2017). doi:10.1242/dev.153056

50. Dasgupta, S., Gupta, K., Zhang, Y., Viasnoff, V. \& Prost, J. Physics of lumen growth. Proc. Natl. Acad. Sci. 115, E4751-E4757 (2018).

51. Miyoshi, H. et al. Prostaglandin E2 promotes intestinal repair through an adaptive cellular response of the epithelium. EMBOJ. 36, 5-24 (2017).

52. Boj, S. F. et al. Forskolin-induced swelling in intestinal organoids: An in vitro assay for assessing drug response in cystic fibrosis patients. J. Vis. Exp. 2017, (2017).

53. Sato, T. et al. Long-term expansion of epithelial organoids from human colon, adenoma, adenocarcinoma, and Barrett's epithelium. Gastroenterology 141, 1762-1772 (2011).

54. Beumer, J. \& Clevers, H. Regulation and plasticity of intestinal stem cells during homeostasis and regeneration. Development 143, 3639-3649 (2016).

55. Bankaitis, E. D., Ha, A., Kuo, C. J. \& Magness, S. T. Reserve Stem Cells in Intestinal Homeostasis and Injury. Gastroenterology 155, 1348-1361 (2018).

56. Ayyaz, A. et al. Single-cell transcriptomes of the regenerating intestine reveal a revival stem cell. Nature 569, 121-125 (2019). 
1 57. Sumigray, K. D., Terwilliger, M. \& Lechler, T. Morphogenesis and Compartmentalization of the Intestinal Crypt. Dev. Cell 45, 183-197.e5 (2018).

3 58. VanDussen, K. L. et al. Development of an enhanced human gastrointestinal epithelial

4 culture system to facilitate patient-based assays. Gut 64, 911-20 (2015).

5 59. Klentzeris, L. D., Bulmer, J. N., Trejdosiewicz, L. K., Morrison, L. \& Cooke, I. D. Infertility: Beta-1 integrin cell adhesion molecules in the endometrium of fertile and infertile women. Hum. Reprod. 8, 1223-1230 (1993).

60. Taylor, C. V., Letarte, M. \& Lye, S. J. The expression of integrins and cadherins in normal human uterus and uterine leiomyomas. Am. J. Obstet. Gynecol. 175, 411-419 (1996).

61. Lessey, B. A. et al. Integrin adhesion molecules in the human endometrium. Correlation with the normal and abnormal menstrual cycle. J. Clin. Invest. 90, 188-195 (1992).

62. Wang, Y. et al. Bioengineered Systems and Designer Matrices That Recapitulate the Intestinal Stem Cell Niche. CMGH 5, 440-453.e1 (2018).

63. Koppes, A. N. et al. Complex, multi-scale small intestinal topography replicated in cellular growth substrates fabricated via chemical vapor deposition of Parylene C. 
a

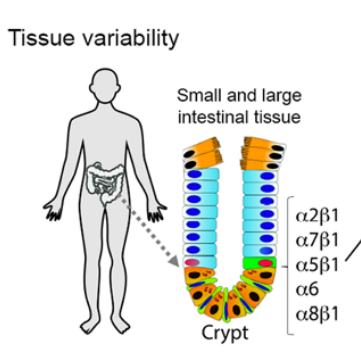

b

b Matrigel
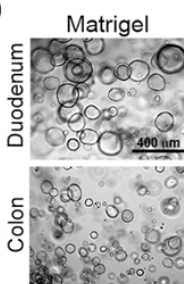

C

\begin{tabular}{|c|c|c|c|c|c|c|}
\hline & & & & & & \\
\hline $\begin{array}{l}\text { Storage } \\
\text { Modulus ( } \mathrm{Pa} \text { ) }\end{array}$ & $620 \pm 20^{\mathrm{a}}$ & $714 \pm 29^{\mathrm{ab}}$ & $817 \pm 63^{\mathrm{bc}}$ & $696 \pm 53^{\mathrm{ab}}$ & $1034 \pm 21^{\mathrm{d}}$ & $929 \pm 63^{\mathrm{cd}}$ \\
\hline Swelling (\%) & $224 \pm 4^{\mathrm{ab}}$ & $195 \pm 5^{\mathrm{bc}}$ & $169 \pm 6^{\mathrm{c}}$ & $296 \pm 18^{\mathrm{a}}$ & $185 \pm 5^{\mathrm{bc}}$ & $196 \pm 12^{\mathrm{bc}}$ \\
\hline Pore Size $(n m)$ & $30 \pm 0.2^{\mathrm{a}}$ & n.d. & $17 \pm 0.5^{b}$ & $33 \pm 1.2^{\mathrm{a}}$ & n.d. & $21 \pm 1.5^{b}$ \\
\hline
\end{tabular}

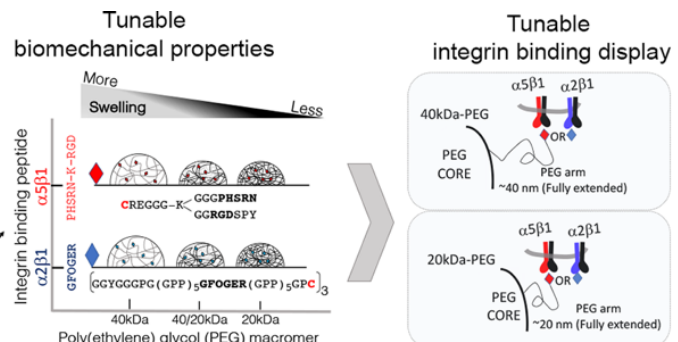

$\begin{array}{cc}40 \mathrm{kDa} & 40 / 20 \mathrm{kDa} \\ \text { Poly(ethylene) } & 20 \mathrm{kDa} \\ \text { glycol (PEG) macromer }\end{array}$

EG-PHSRN-K-RGD ( $\alpha 5 \beta 1)$

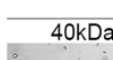

PEG-GFOGER $(\alpha 2 \beta 1)$

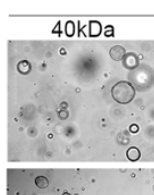

\begin{tabular}{c}
$40 / 20 \mathrm{kDa}$ \\
\hline
\end{tabular}
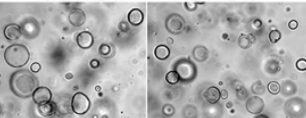

$\because: 0$

Fig 1| Synthetic matrices support enteroid formation from single cells. a, Niche-inspired synthetic matrix design. b, Representative images of six-day old duodenal and colon enteroids emerging in the synthetic matrices. Seven donors (six human duodenal, one colon, and one mouse colon) were evaluated (Extended Data Fig 2). Scale bar: $400 \mu \mathrm{m}$. c, Biomechanical properties of the synthetic matrices. Storage modulus data $(n=6$ gels). Swelling and theoretical pore size data $(\mathrm{n}=12$ gels). d, Enteroid diameter of duodenal ( $\mathrm{n}=3$ experiments) and colon ( $\mathrm{n}=2$ experiments) donors. The number of enteroids measured is depicted under each violin plot. Matrigel diameters were significantly bigger $(\mathrm{P}<0.0001)$. e, Enteroid formation efficiency of duodenunal and colon donors from two independent experiments. Each symbol represents a single hydrogel with the mean and SEM. 
a
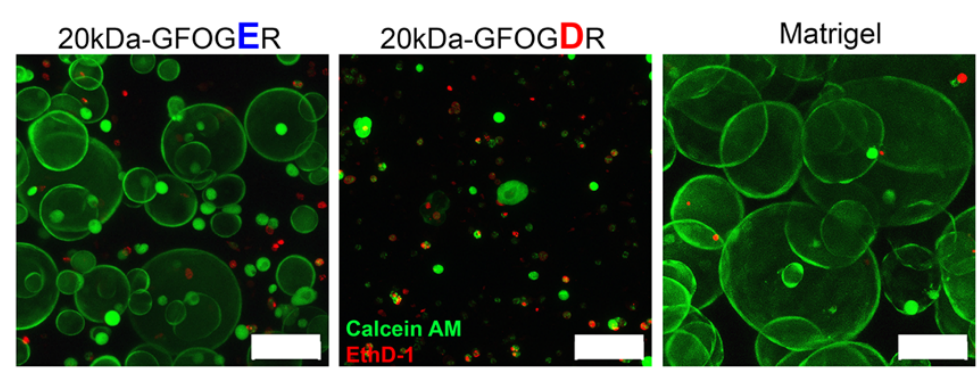

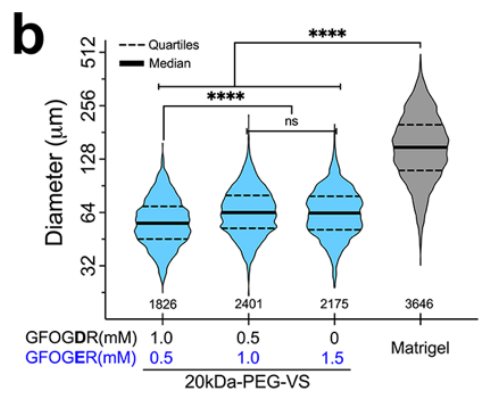

C

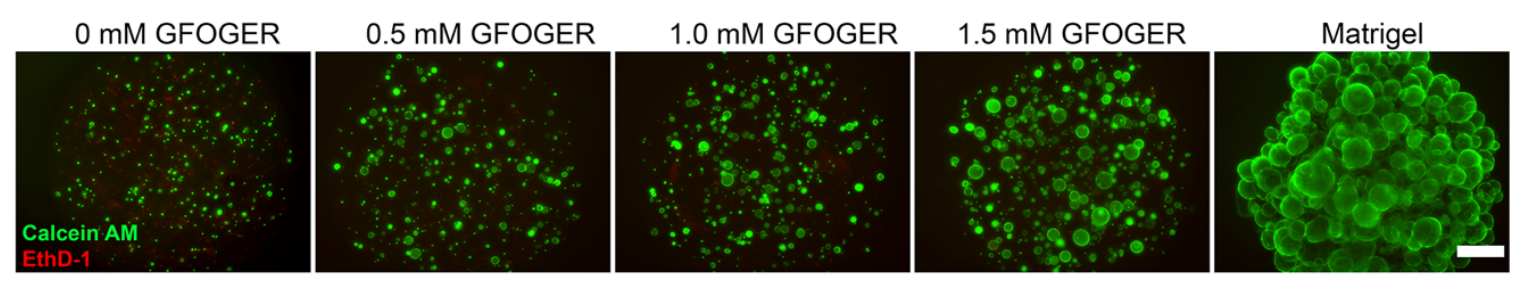

d

Matrigel

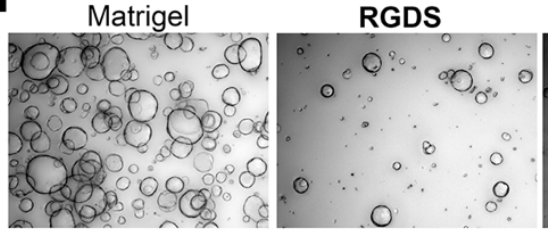

e
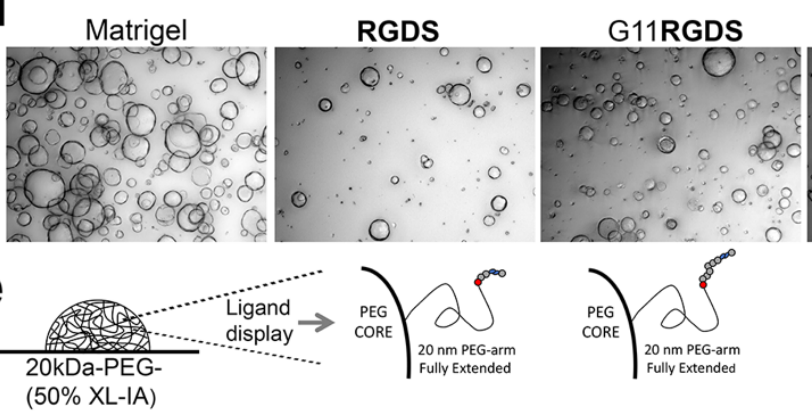

f

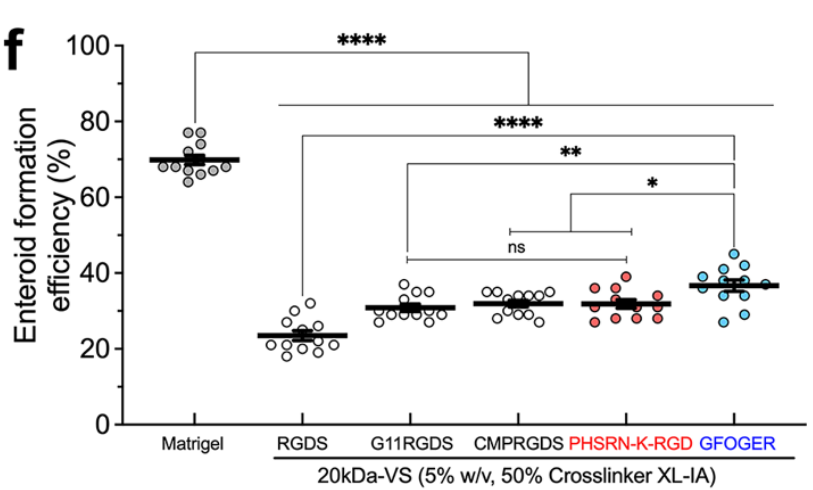

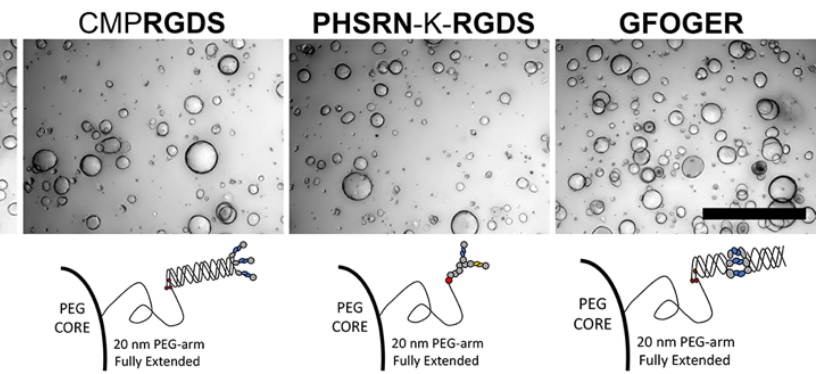

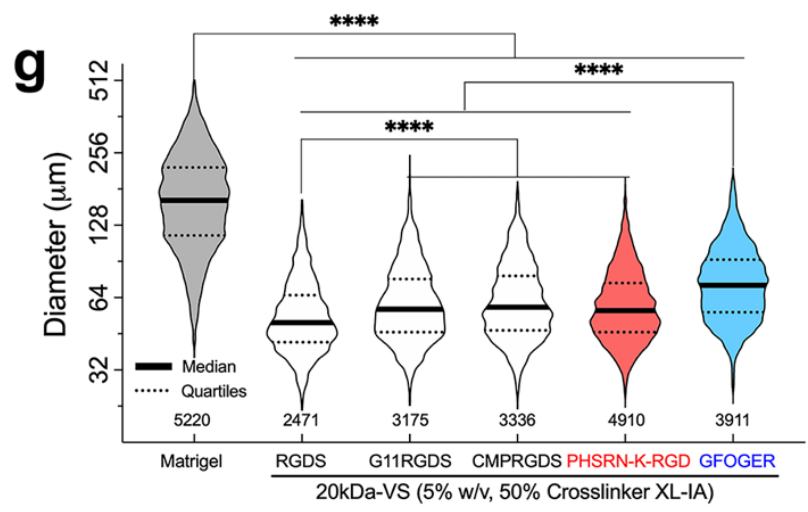

Fig $2 \mid$ Robust enteroid growth depends on the $\alpha 2 \beta 1$ integrin binding peptide, GFOGER. a, An aminoacidic point-mutation in GFOGER $(E \rightarrow D)$ abolishes enteroid formation. Live-dead 3D projections of enteroids. b-c, GFOGER dose response effect on $\mathbf{b}$, enteroid diameter and $\mathbf{c}$, viability. d-f, Effect of the $\alpha 5 \beta 1$ ligand presentation on enteroid formation efficiency and diameter. d, Enteroids in matrices with various ligand presentation in e. f, Enteroid formation efficiency from two experiments. Each symbol represents a single hydrogel with the mean and SEM. g, Enteroid diameters from two independent experiments. The number of enteroids measured per matrix condition in $\mathbf{b}$ and $\mathbf{g}$ is depicted under each violin plot. Scale bar in $\mathbf{a}: 100$ $\mu \mathrm{m}$, c-d: $500 \mu \mathrm{m}$. Six-day old enteroids were used/analyzed in a-d and $\mathbf{g}$. 

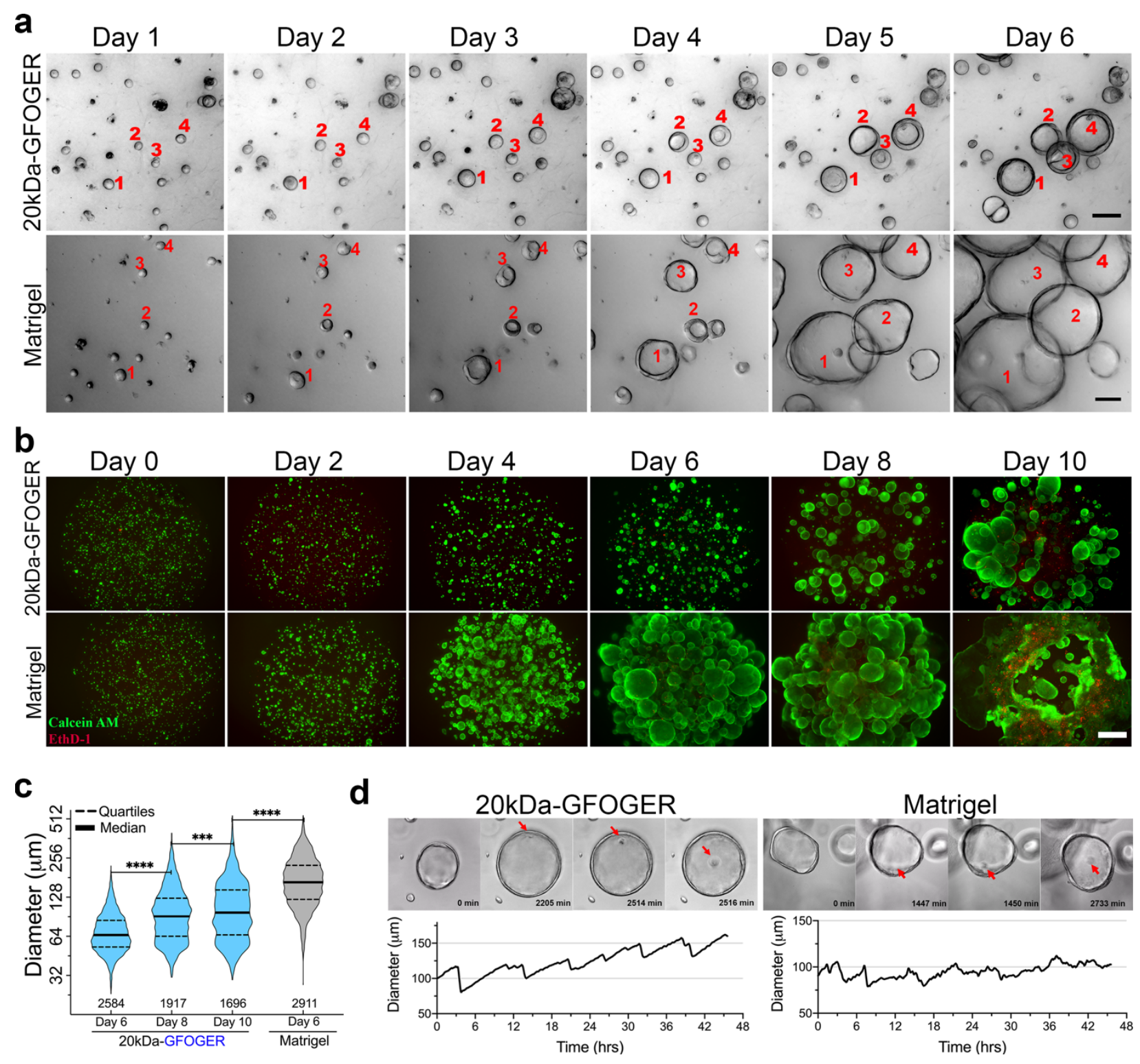

Fig 3| Single cell proliferation and enteroid formation in the synthetic ECM are similar to those in Matrigel. a, Time-course imaging of enteroids emerging from single cells. Scale bar: $50 \mu \mathrm{m}$. b, Live-dead 3D projections of enteroids over ten days. Scale bar: $500 \mu \mathrm{m}$. c, Timecourse enteroid diameter ( $\mathrm{n}=2$ experiments). The number of enteroids measured is depicted under each violin plot. d, Time-lapsed imaging analysis of changes in diameter of a single enteroid from days 4 to 6 . Red arrows point to single cells from the epithelial layer being extruded into the lumen. 
a

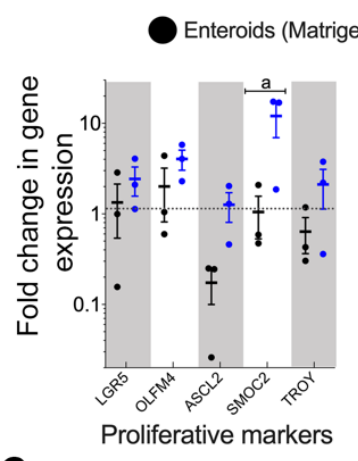

C
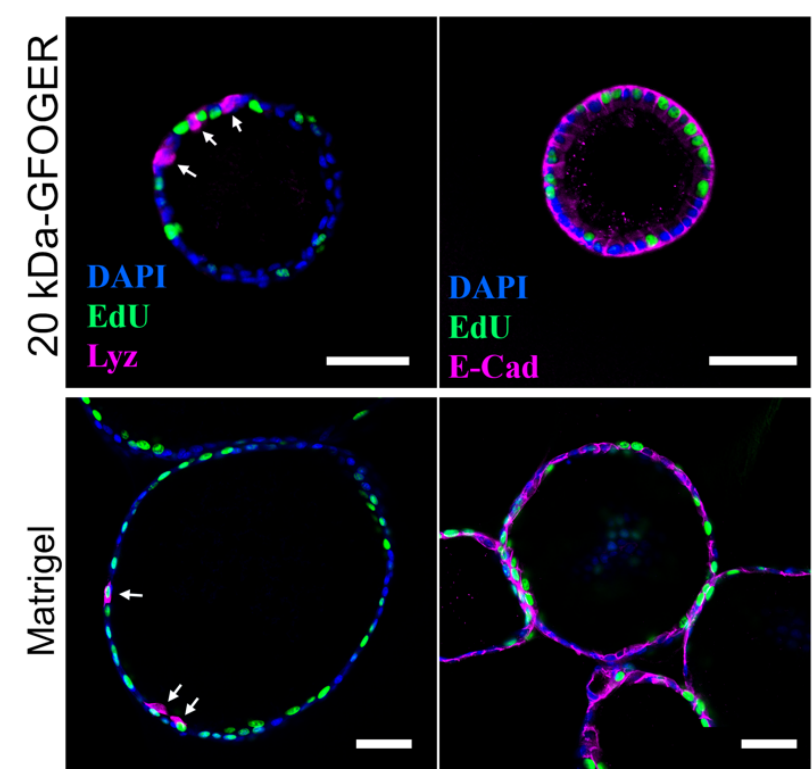

Enteroids (20kDa-GFOGER)

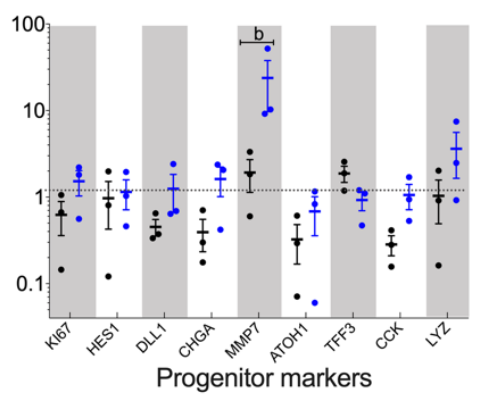

Progenitor markers
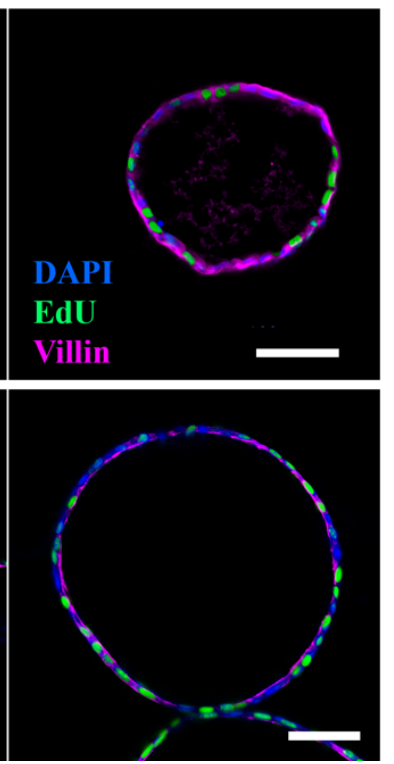

b

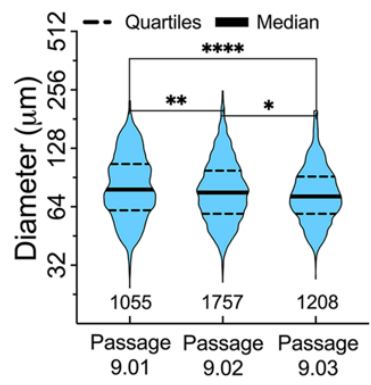

d

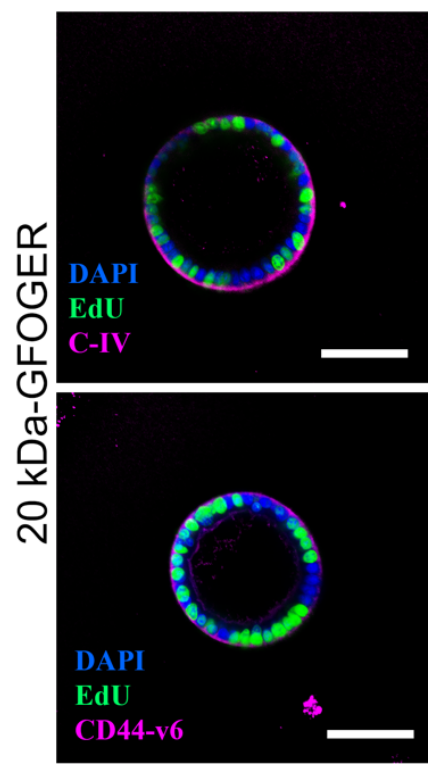

Fig 4| Enteroids in the synthetic ECM show diverse cellular composition. a, Fold change in gene expression of enteroids in the synthetic ECM or Matrigel. Data from three independent experiments. b, Quantification of enteroid diameters from three successive passages in the synthetic ECM. Data from a single experiment with 12 replicates in each passage. The number of enteroids measured is depicted under each violin plot. c-d, Immunostaining analysis of six-day old enteroids in the synthetic ECM and Matrigel showing proliferative cells (Edu), Paneth cells (Lyz), epithelial markers (E-cad and Villin) and basolateral markers (C-IV and CD44-v6). Scale bar: $50 \mu \mathrm{m}$. Six-day old enteroids were used in all panels. 
a

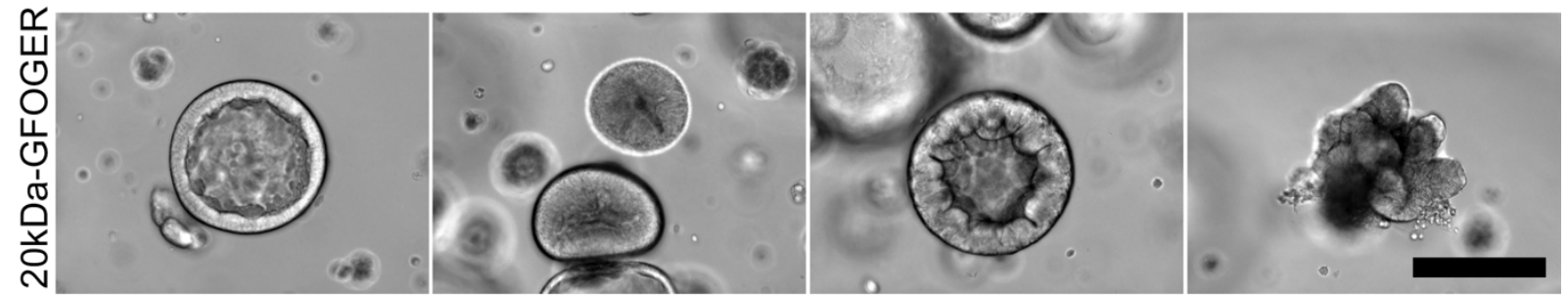

b

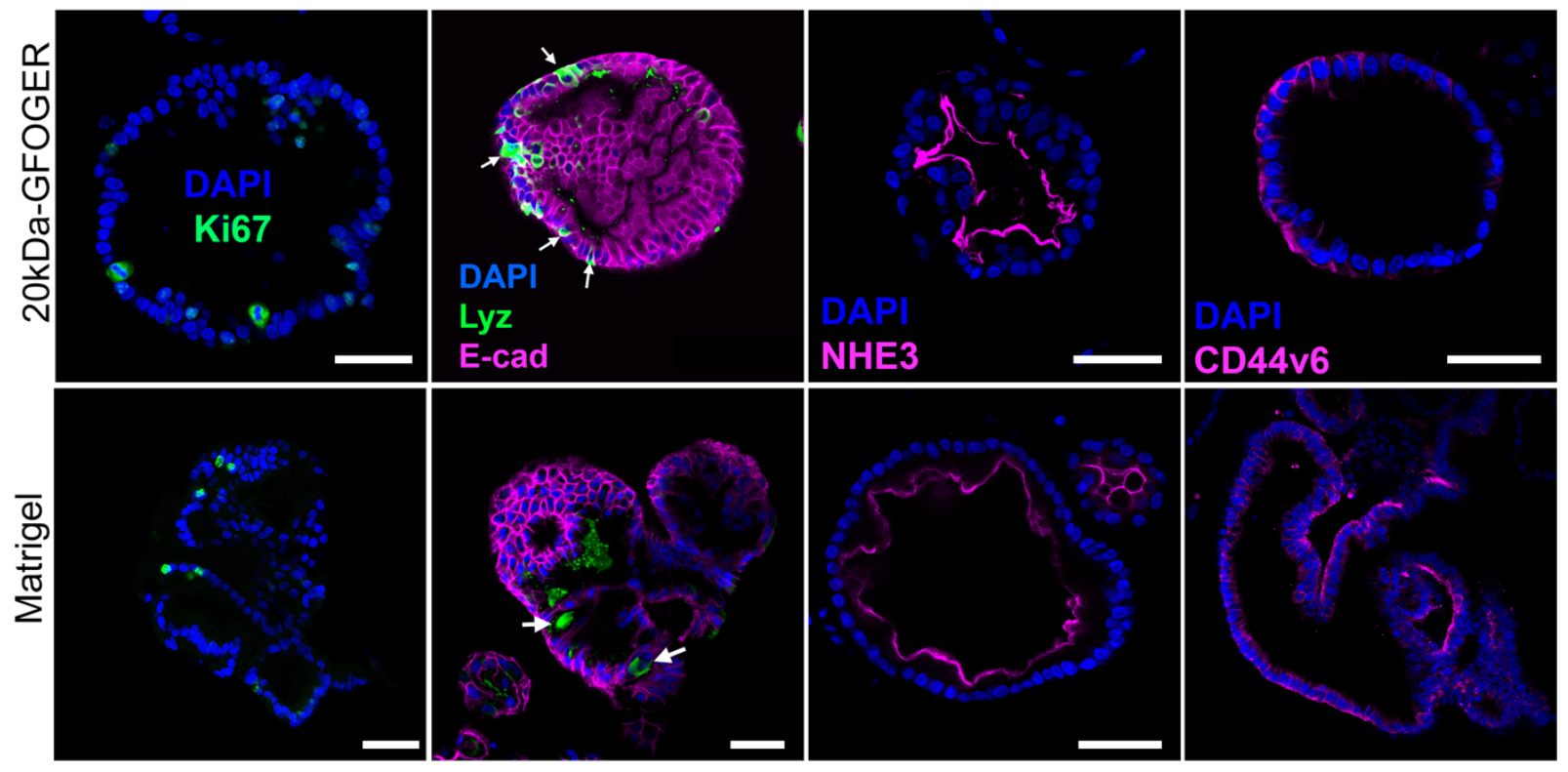

C
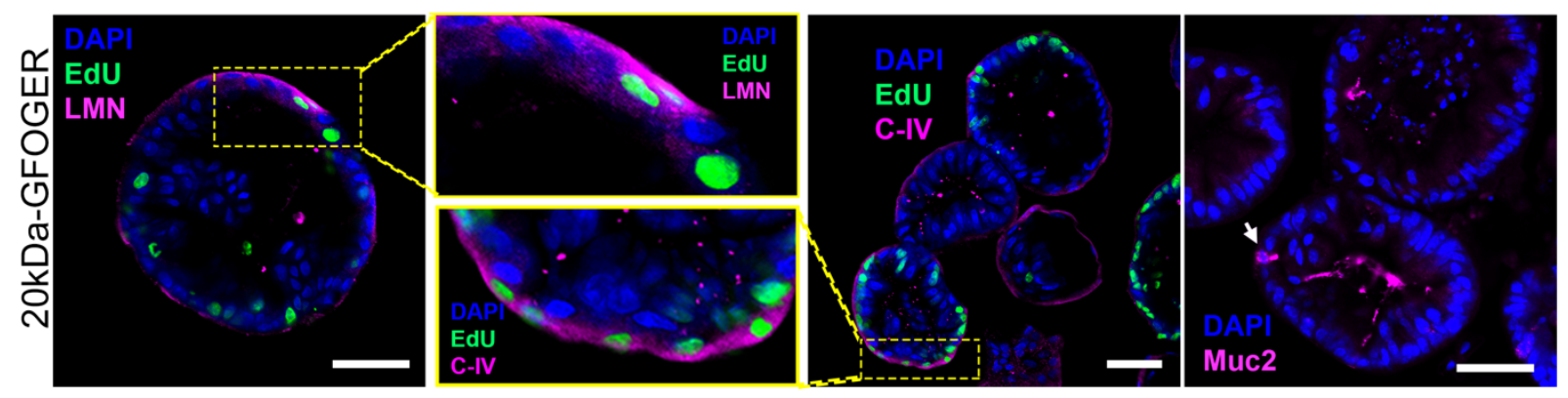

Fig 5| Enteroids in the synthetic ECM undergo differentiation. a, Bright-field images of 10day old human enteroids growing in the synthetic ECM showing diverse phenotypes upon inducing differentiation. Scale bar: $200 \mu \mathrm{m}$. b-c, Immunostaining analysis of 10-day old organoids in the synthetic ECM and Matrigel showing proliferative cells (Ki67), Paneth cells (Lyz), Goblet cells (Muc2). Epithelial marker (E-cad), apical marker (NHE3) and basolateral markers (CD44v6, CIV, and LMN). Scale bar: $500 \mu \mathrm{m}$. 
a

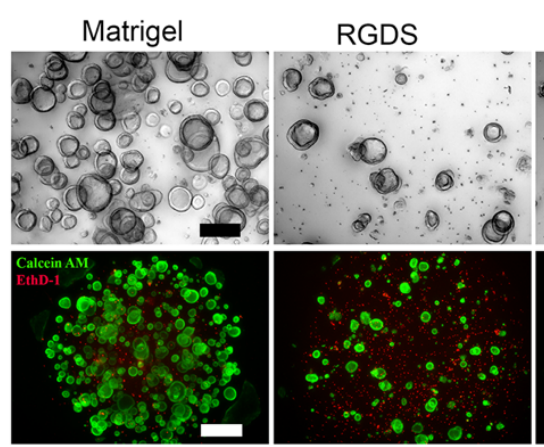

b

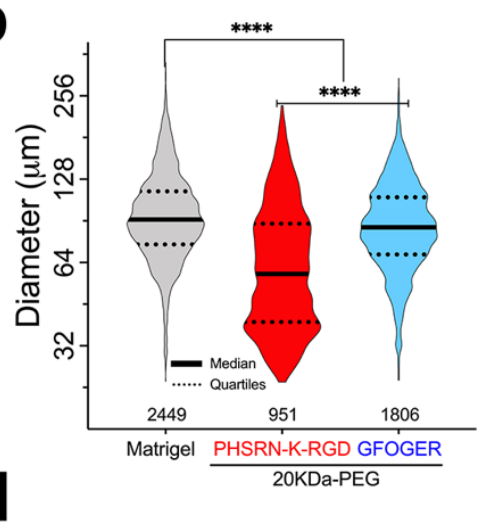

C
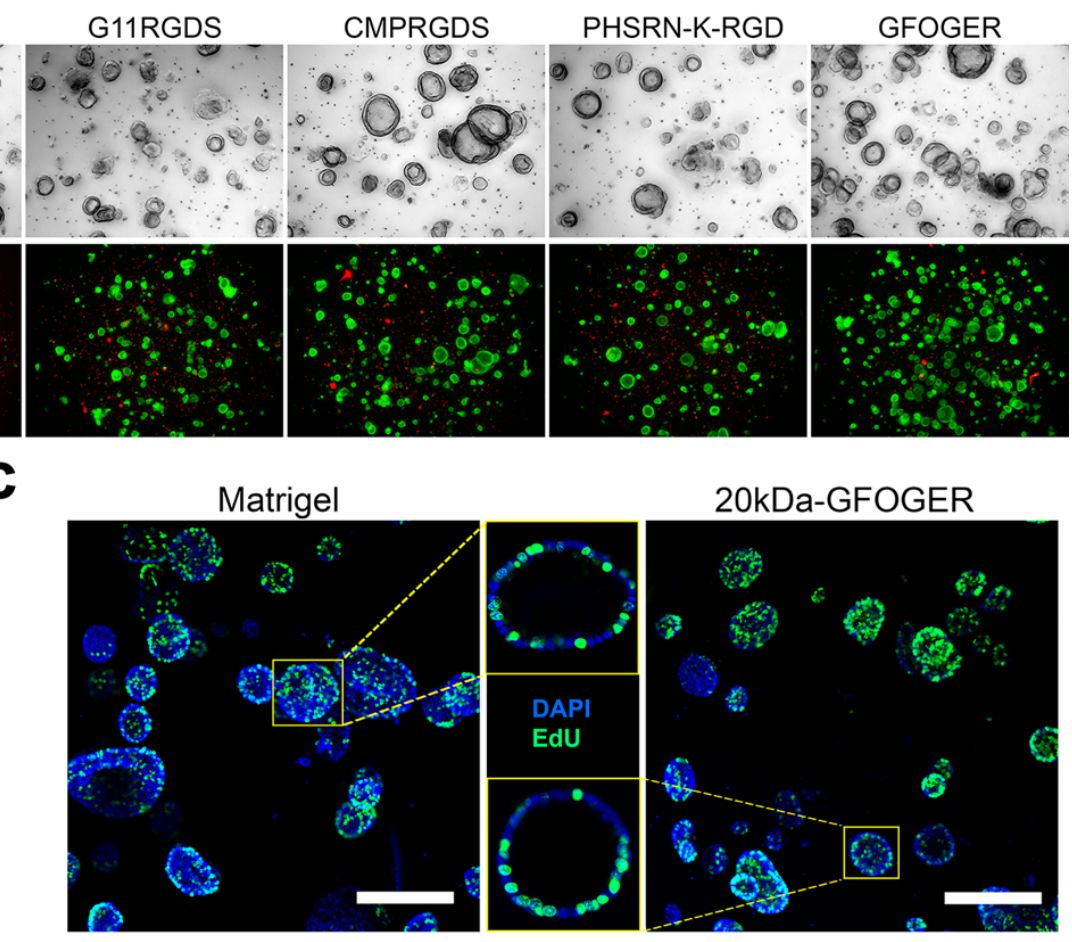

20kDa-GFOGER

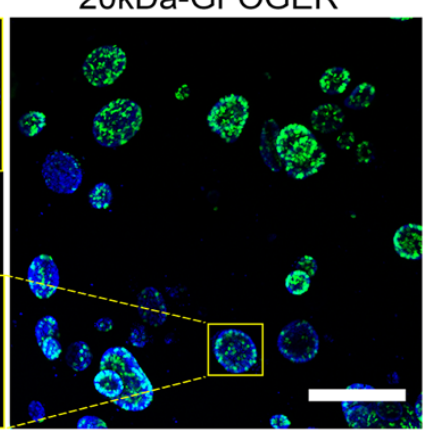

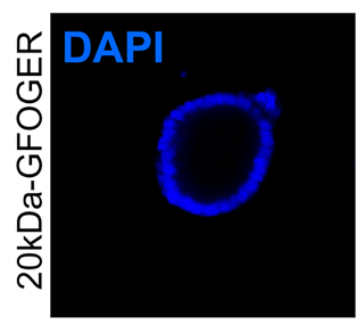
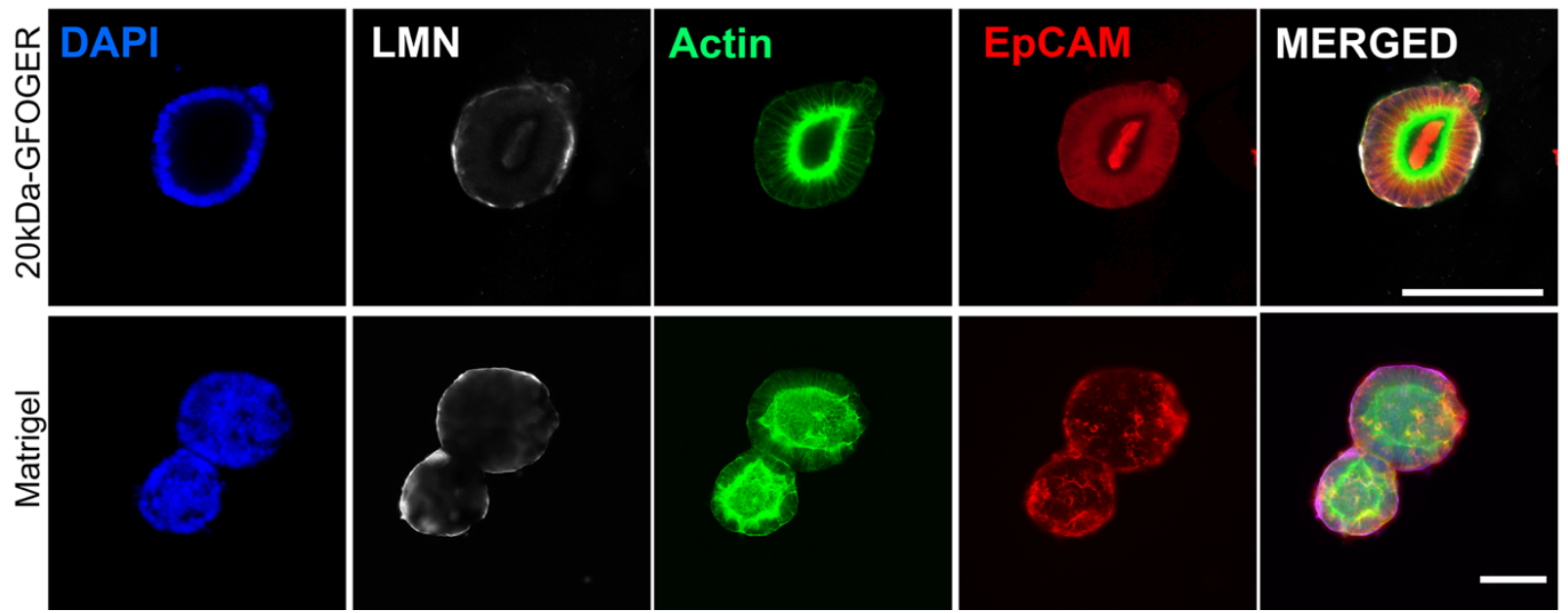

Figure 6| Human endometrial organoids can be cultured in a synthetic ECM. a, Top, Bright field images of endometrial organoids in the synthetic matrix and Matrigel, eight days after encapsulation. Bottom, live-dead 3D projections of endometrial organoids in the indicated matrix after eight days of culture. Scale bar top: $200 \mu \mathrm{m}$, bottom: $500 \mu \mathrm{m}$. b. Diameter of eigh-day old endometrial organoids ( $\mathrm{n}=2$ independent experiments). The number of organoids measured per matrix condition is depicted under each violin plot. c-d, Immunostaining analysis of organoids showing proliferative cells (Edu), apical markers (actin), epithelial marker (EpCAM), and basolateral marker $(\mathrm{LMN})$. Scale bar in $\mathbf{c}$ and $\mathbf{d}$, bottom panel $=200 \mu \mathrm{m}$, top panel in $\mathbf{d}$ : $100 \mu \mathrm{m}$. 


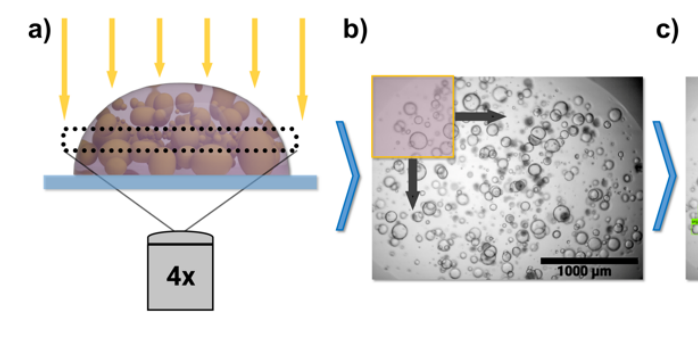

c)

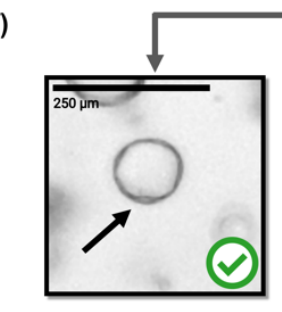

Individual

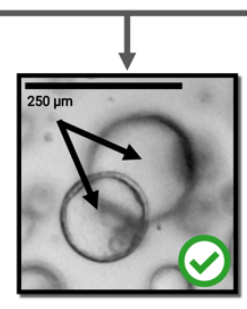

Partial Overlap

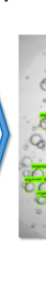

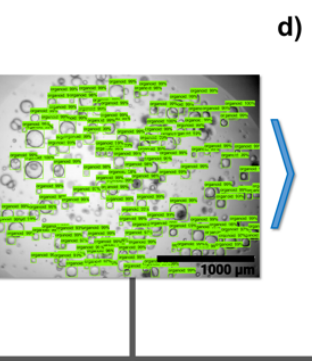

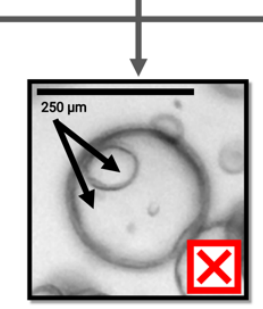

Full Overlap d)

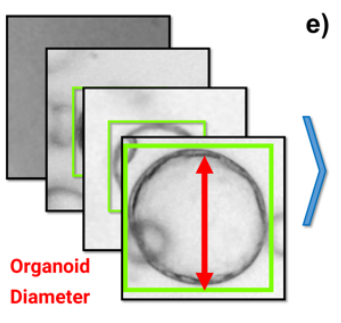

e)

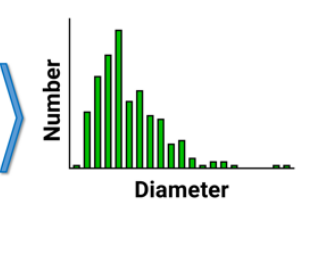

Extended Data Fig 1| Enteroid diameter measurements using Orgaquant, a deep convolutional neural network analysis platform. a, A single plane, approximately in the middle of the matrix droplet, is imaged in bright-field (BF) using a 4x objective. $\mathbf{b}$, The BF image is sectioned into smaller images using a sliding window of 450 pixels, with an overlap window of 200 pixels. The overlapping allows to capture organoids that otherwise would be at the edge of the slide window. The algorithm also eliminates redundant bounding boxes that might occur due to overlapping. c, A representative BF image showing bounding boxes around the enteroids measured. $\mathbf{d}$, The diameter of the bounding boxes is used to calculate the diameter of the enteroids in focus, which are then presented as a distribution plot, as in e. f, The convolutional network was trained to ignore enteroids that have full overlap, are out of focus, or appear as single cells. 
a
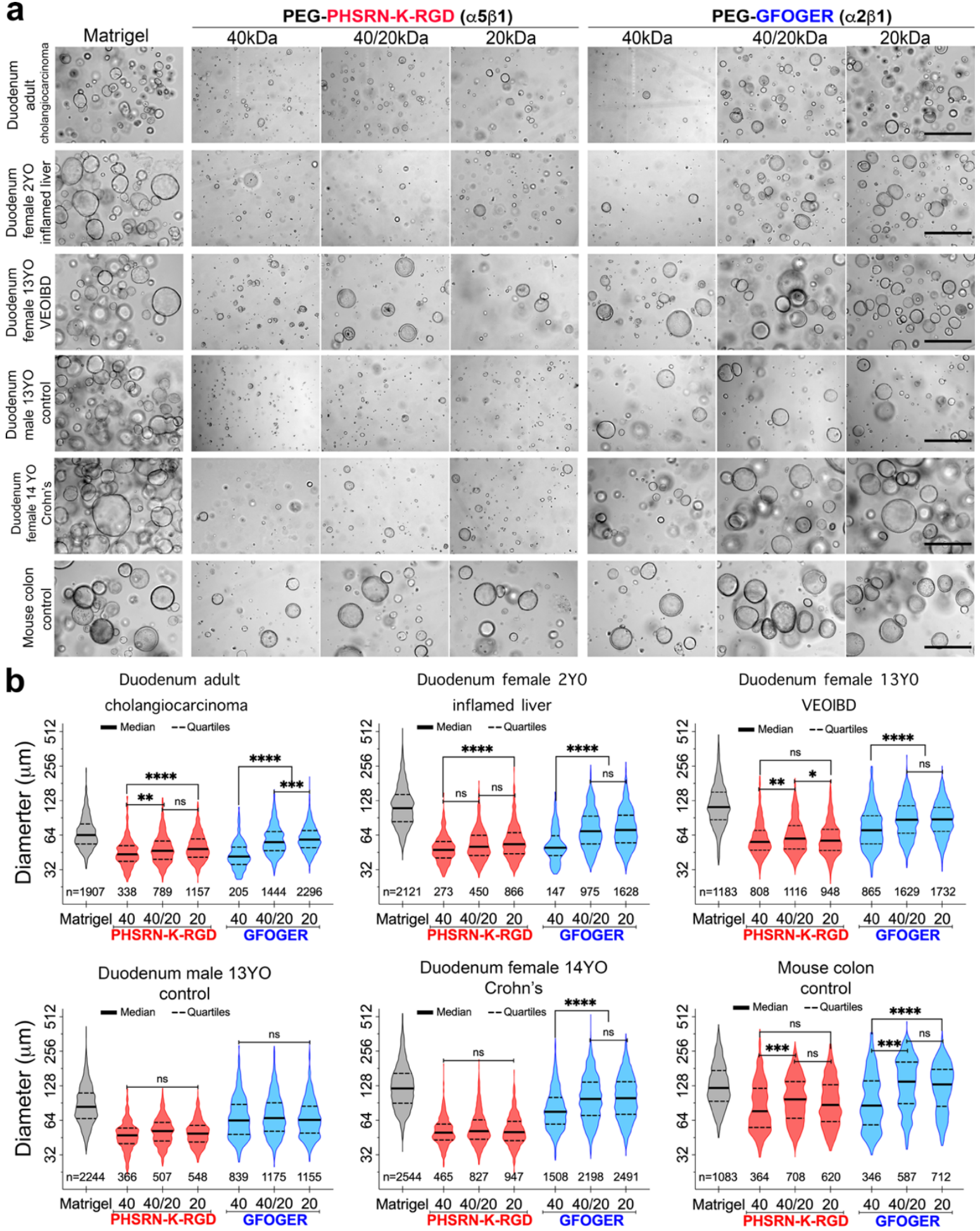

Extended Data Fig 2| Synthetic matrices support enteroid formation from multiple donors and tissue types. a, Representative images of six-day (human) and four-day (mouse) enteroids growing in the synthetic matrices. Scale bars: $400 \mu \mathrm{m}$. b, Quantification of enteroid diameter from three independent experiments for human donors and two independent experiments for mouse donor. The number of enteroids measured per matrix condition and donor is depicted under each violin plot. Matrigel diameters were significantly bigger $(\mathrm{P}<0.0001)$. 
a

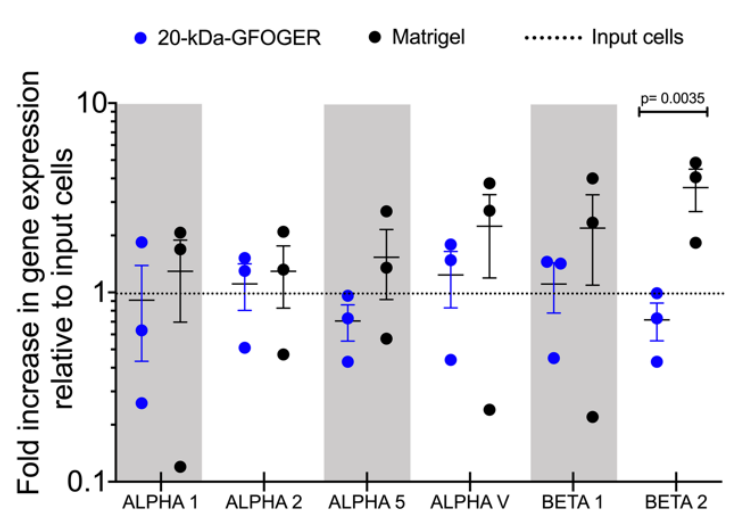

b

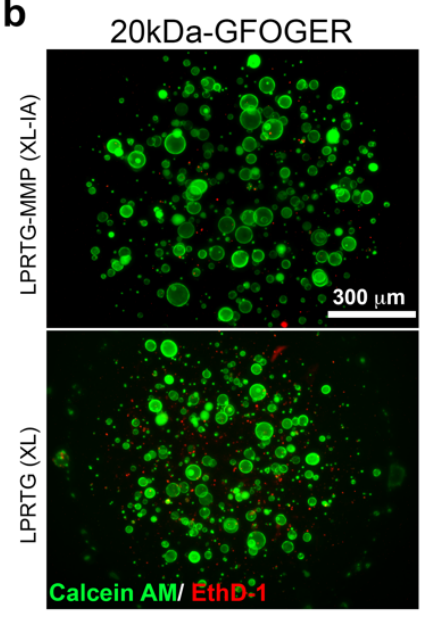

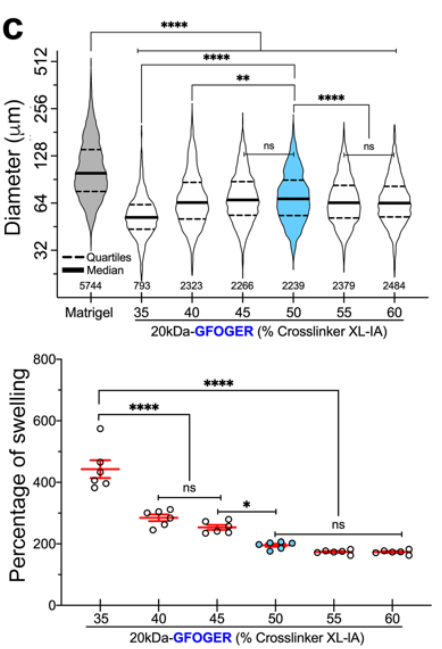

Extended Data Fig 3| Integrin expression and matrix's biomechanical properties affect enteroid formation. a, Integrin expression profile of six-day old human duodenal enteroids. b, Live/dead 3D projections of six-day old enteroids growing in matrices made with a degradable (LPRTG-MMP) and non-degradable (LPRTG) crosslinker. c, Effect of crosslinker density (LPRTG-MMP) on the enteroid diameter (top) and gel swelling property (bottom). Data from three independent experiments. The number of six-day old enteroids measured is depicted under each violin plot in the top panel. Bottom panel, each symbol represents a single hydrogel with the mean and SEM. 
a

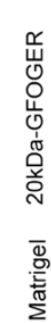

Day 1
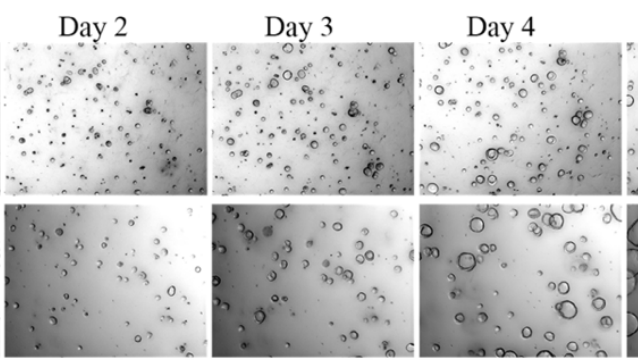

b

Human duodenum

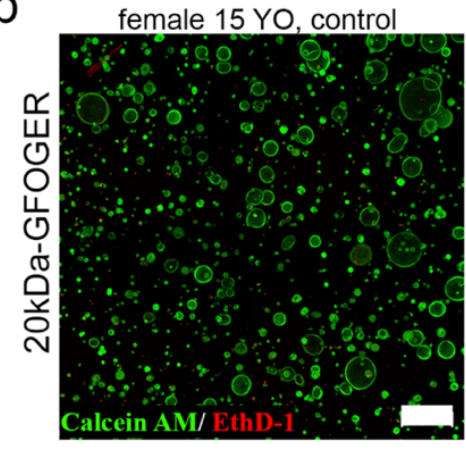

Human duodenum female 14 YO, Crohn's
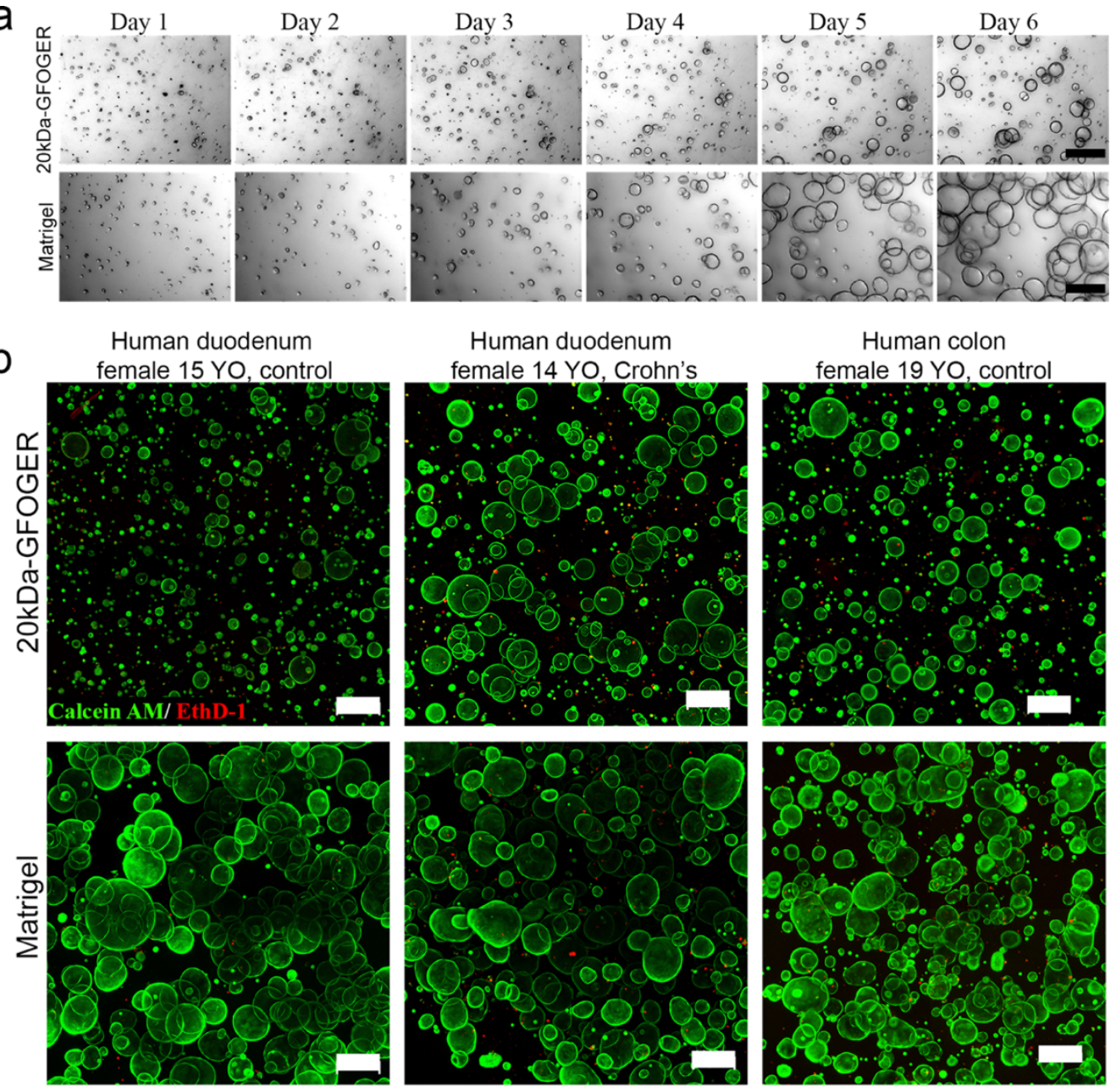

C

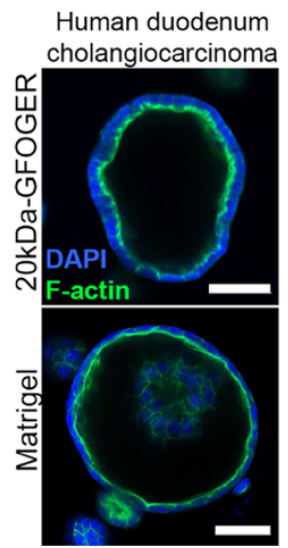

Human duodenum
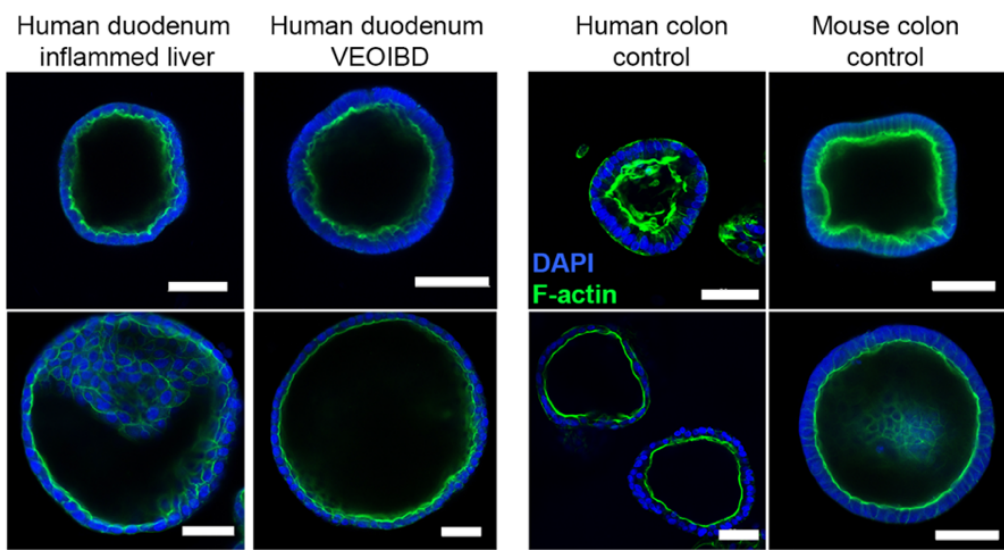

Extended Data Fig 4| Single cell proliferation and enteroid formation in the synthetic ECM are similar to those in Matrigel. a, Time-course imaging of single cells forming enteroids in the 20kDa-GFOGER and Matrigel. Scale bar: $50 \mu \mathrm{m}$. b. Live-dead 3D projections of six-day (duodenal) and eight-day (colon) enteroids in the 20kDa-GFOGER and Matrigel. Scale bar: 500 $\mu \mathrm{m}$. c, Human duodenal (left) and colon enteroids display well-defined and polarized lumens when grown in the synthetic ECM or Matrigel. Human duodenal enteroids were grown for six days, human colon for eight days, and mouse colon for four days. 
a

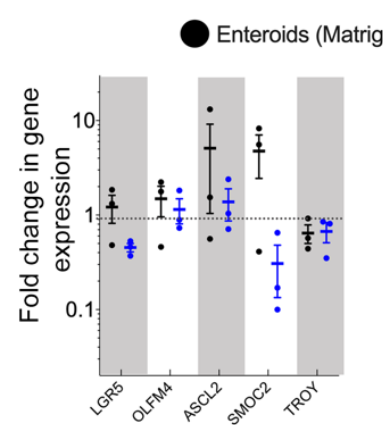

C b

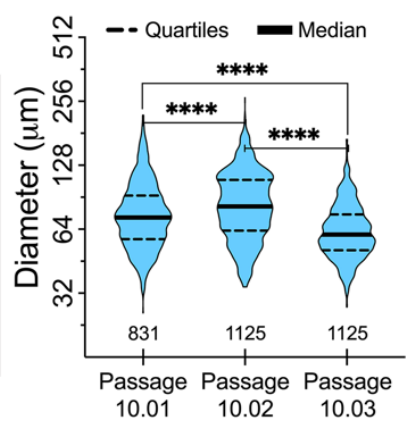

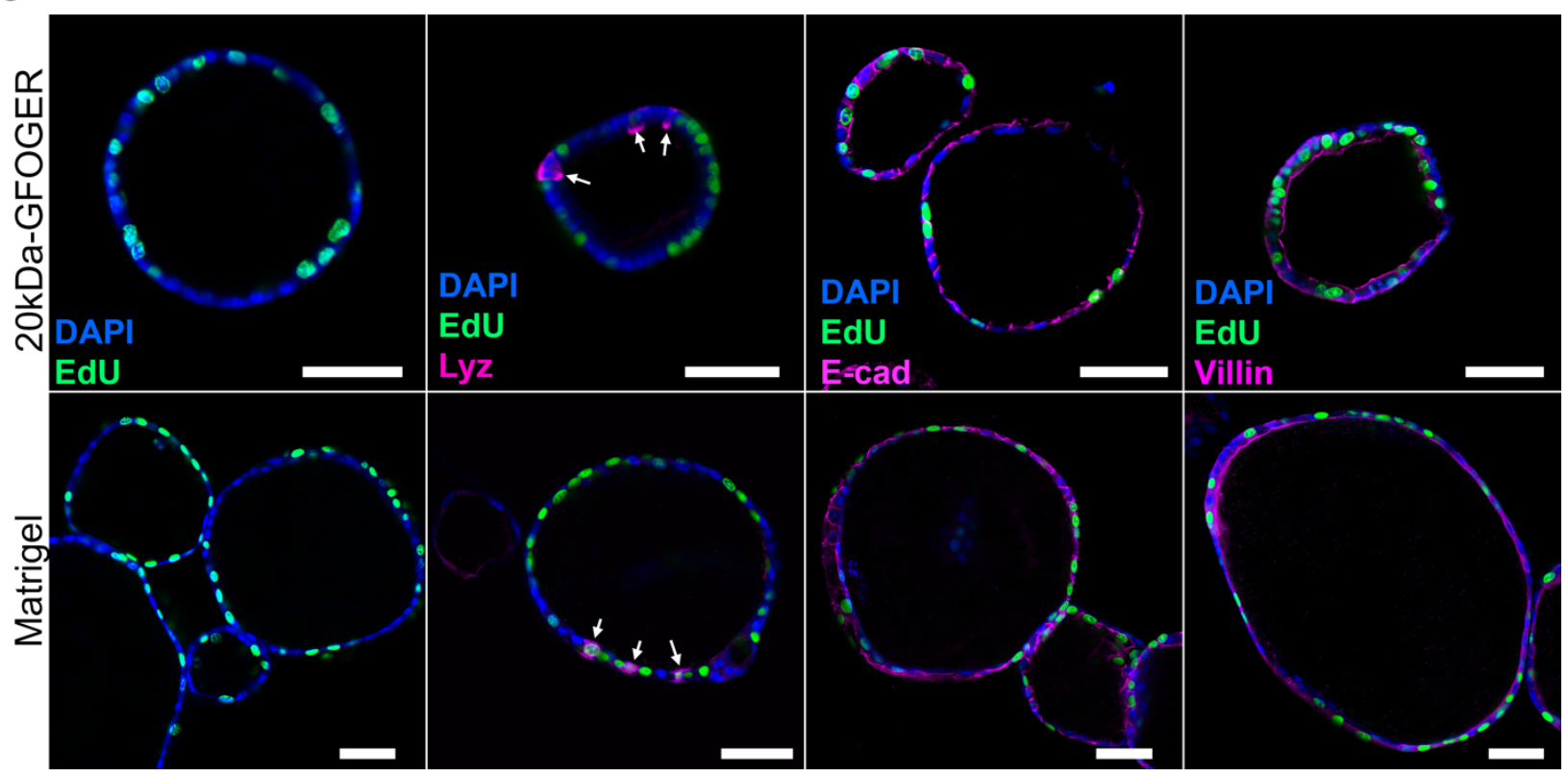

Extended Data Fig 5| Human duodenal enteroids from a Crohn's disease donor show correct cellular composition in the synthetic ECM. a, Fold change in gene expression of six-day old enteroids in the synthetic ECM and Matrigel relative to the input cells. Data from three independent experiments. b, Quantification of six-day old enteroid diameters from three successive passages in the synthetic ECM. Data from a single experiment with 12 replicates per consecutive passage. The number of enteroids measured is depicted under each violin plot. c, Immunostaining analysis of sixday old enteroids in the synthetic ECM and Matrigel showing proliferative cells (Edu), Paneth cells (Lyz) and epithelial markers (E-cad and Villin). 
a

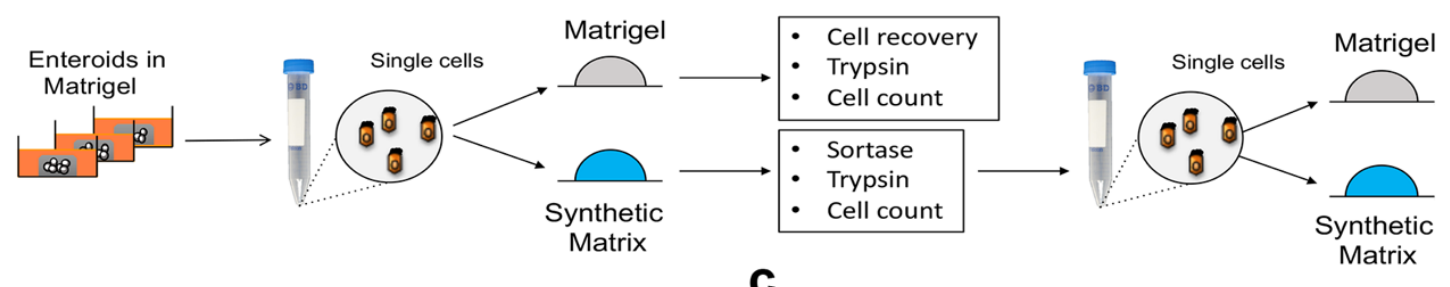

b

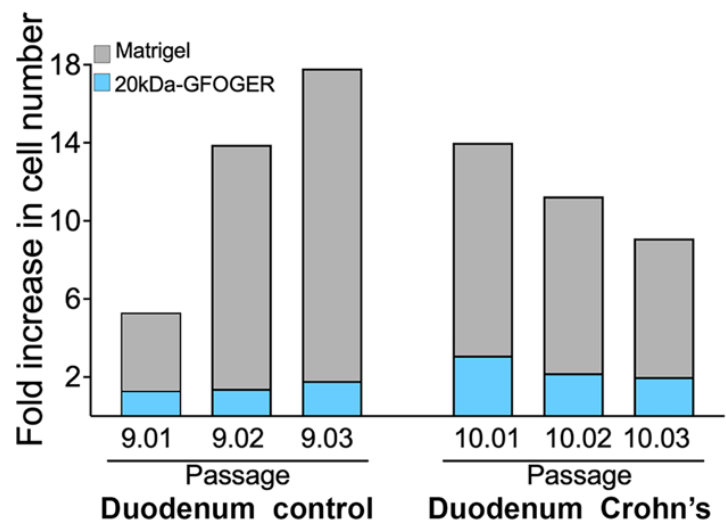

\section{d}

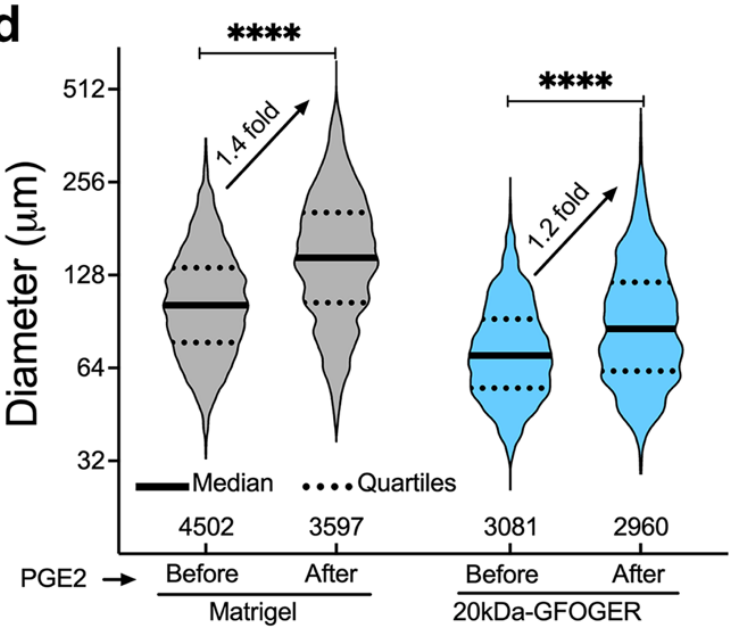

C

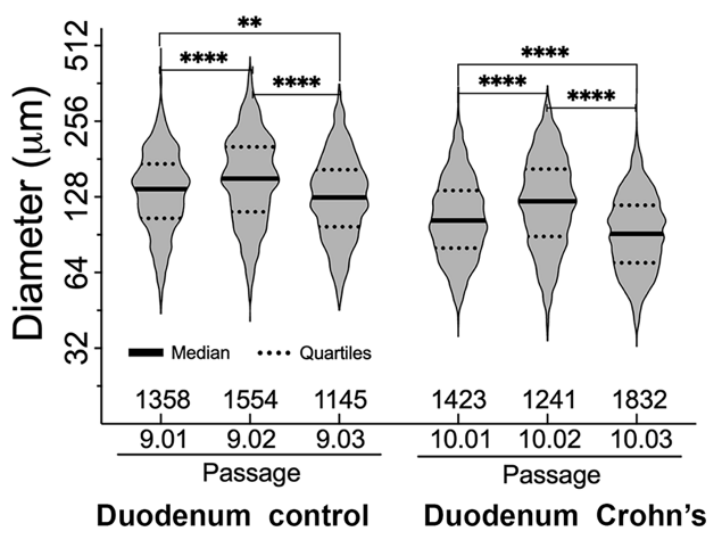

e

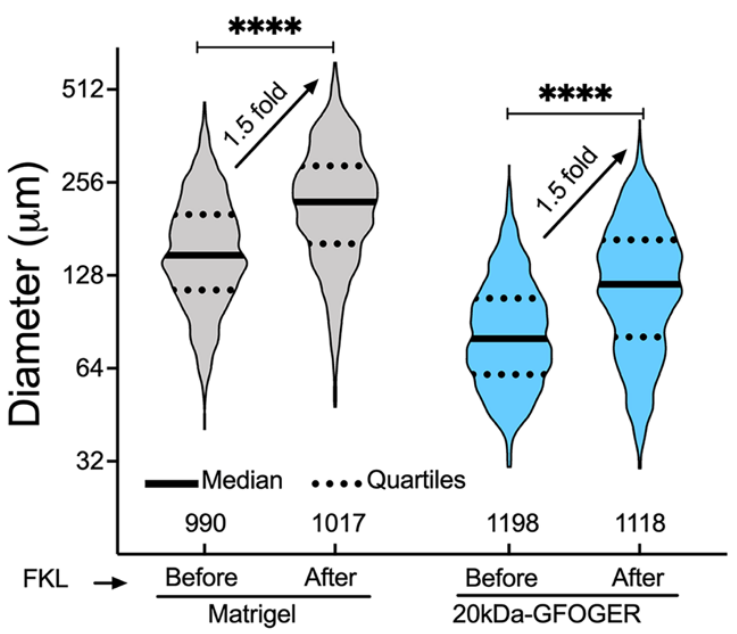

Extended Data Fig 6| Enteroids grown in the synthetic ECM retain their proliferative capacity and respond to basal stimulation. a, Overall strategy to test the proliferative capacity of enteroids grown in the synthetic ECM. After Sortase-mediated dissolution, six-day old enteroids were digested and single cells re-embedded in new synthetic ECM or Matrigel. b, Fold increase in cell number after six days of culture in each passage from two human donors. Data represent three consecutive passages using enteroids collected from 24 to 48 individual hydrogels pooled. c, Diameter of six-day old enteroids in Matrigel emerging from enteroids previously grown in the synthetic ECM. Data from a single experiment with three consecutive passages. The number of enteroids measured is depicted under each violin plot. d-e, Diameter of enteroids before (day six) and after (day seven) stimulation with PGE2 $(n=3)$ and forskolin $(n=2)$. The number of enteroids measured is depicted under each violing plot. 

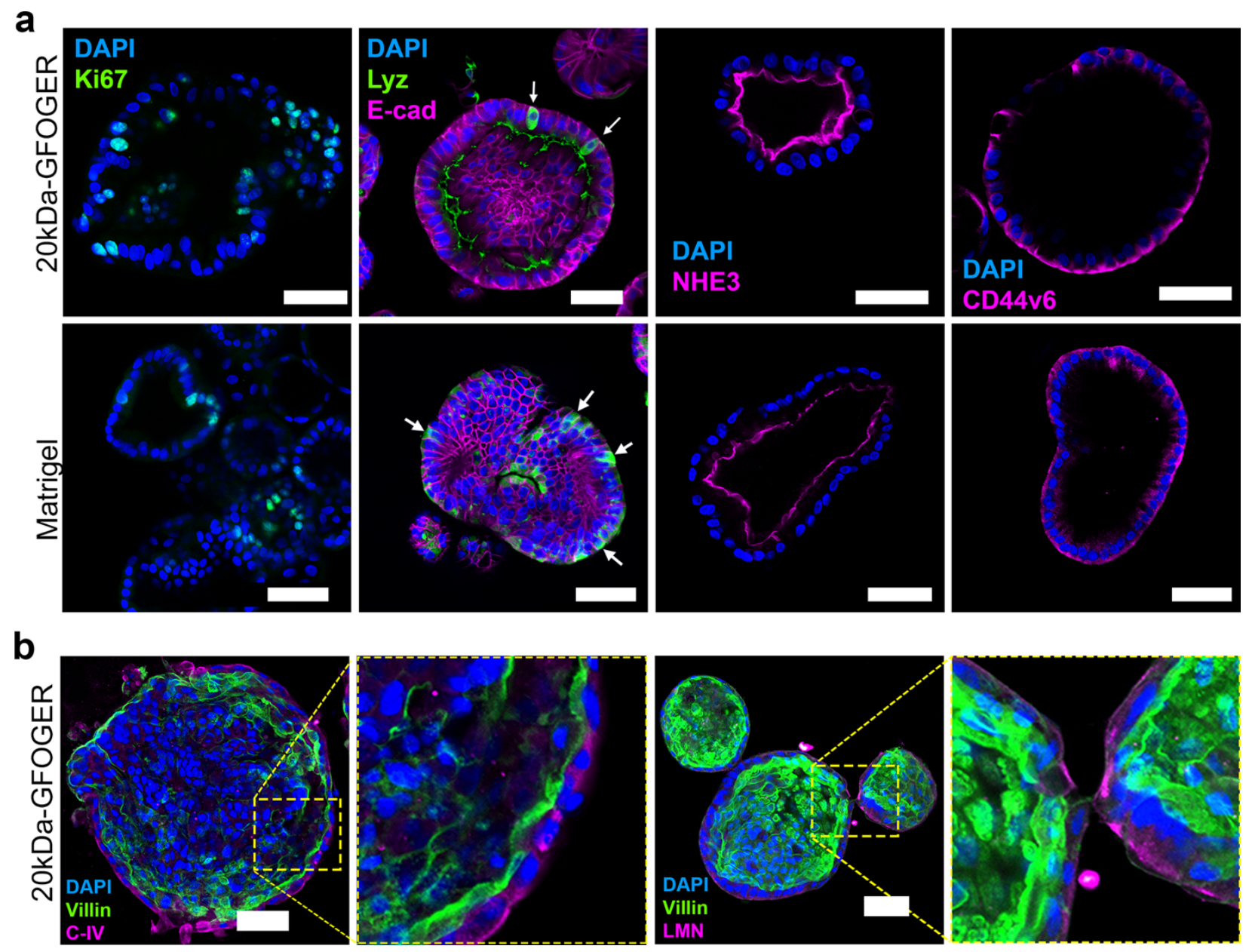

Extended Data Fig 7| Human duodenal enteroids from a Crohn's disease donor show appropriate cellular composition after differentiation. a-b, Immunostaining analysis of 10-day old organoids differentiated in the synthetic ECM and Matrigel showing proliferative cells (Ki67), Paneth cells (Lyz), epithelial marker (E-cad), apical markers (NHE3, Villin) and basolateral markers (CD44v6, C-IV, and LMN). Scale bar: $50 \mu \mathrm{m}$. 

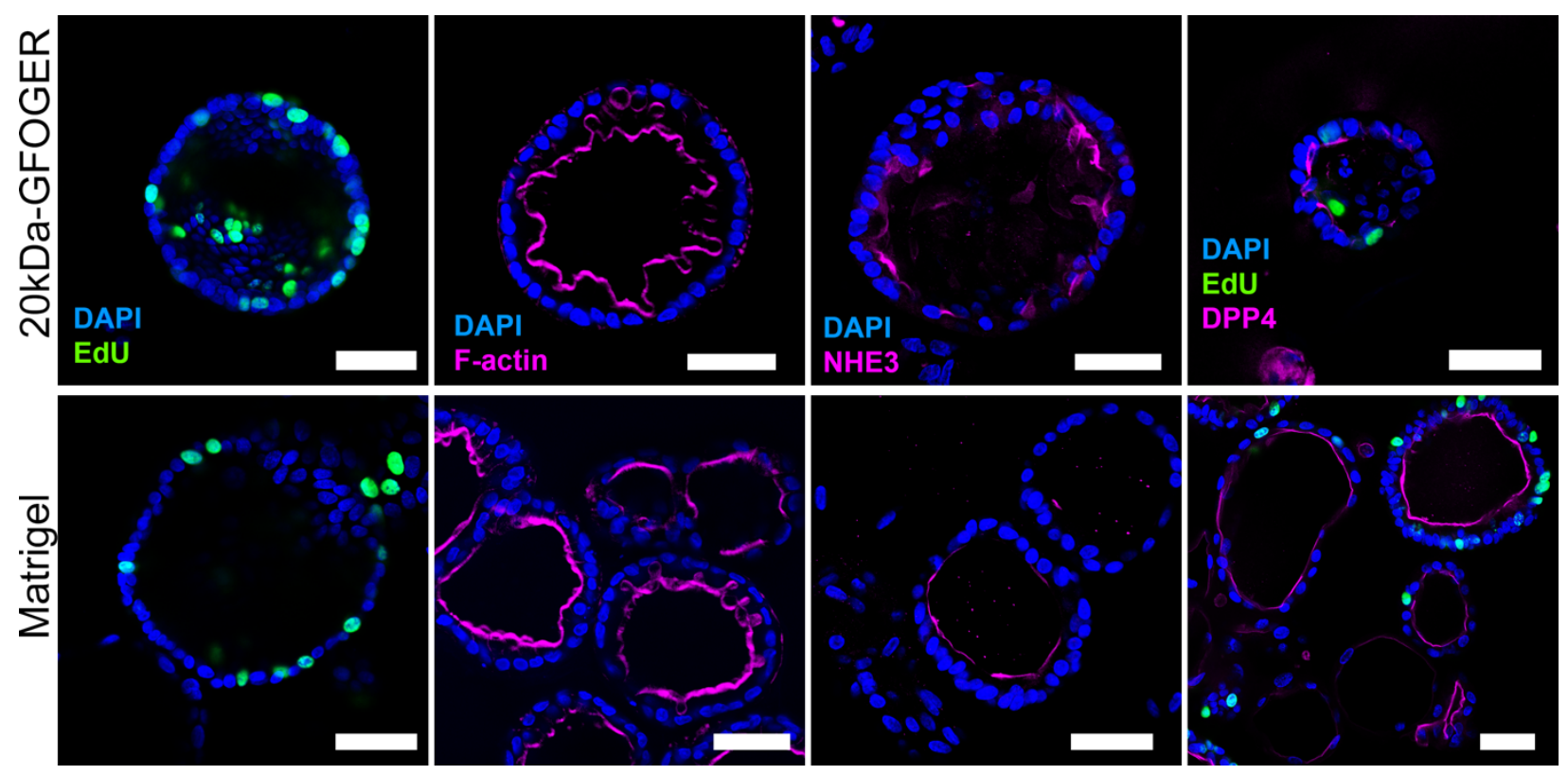

Extended Data Fig 8| Human colon organoids in the synthetic ECM exhibit correct architecture and cellular composition. Immunostaining analysis of 10-day old organoids emerging in the synthetic matrix and Matrigel showing proliferative cells (Edu), and correct localization of apical markers (F-actin, NHE3 and DPP4).

a

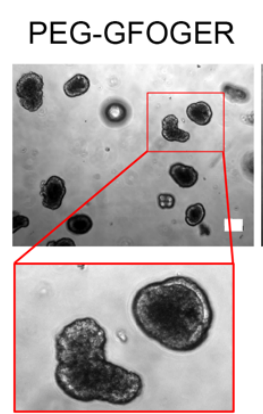

b

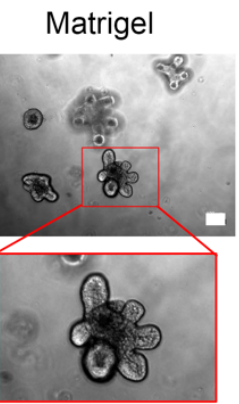

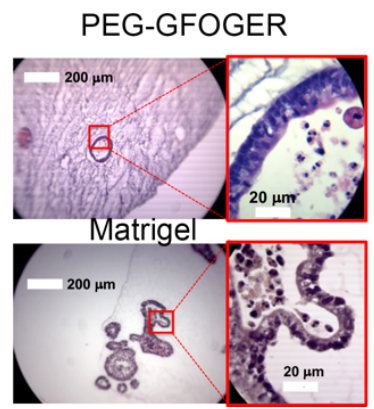

C

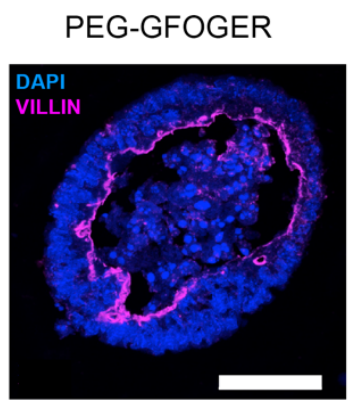

Matrigel

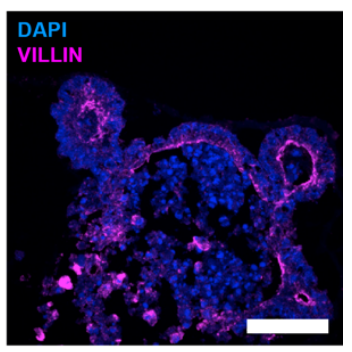

Extended data Fig 9| Mouse small intestinal organoids, in the synthetic ECM, undergo differentiation. a, Brightfield images of five-day old enteroids undergoing differentiation. Scale bar: $100 \mu \mathrm{m}$. b. Histological sections showing accumulation of apoptotic cells in the lumen upon differentiation. $\mathbf{c}$, Immunostaining of differentiated organoids showing apical villin marker. 


\section{Supplementary Data}

Table 1: List of Taqman probes used for enteroid characterization

\begin{tabular}{ll|l} 
Name & Gene ID & Length (nt) \\
\hline GADPH & Hs02786624_g1 & 157 \\
\hline LGR5 & Hs00969422_m1 & 61 \\
\hline OLFM4 & Hs00197437_m1 & 85 \\
\hline ASCL2 & Hs00270888_s1 & 101 \\
\hline SMOC2 & Hs01591663_m1 & 80 \\
\hline TROY & Hs00218634_m1 & 59 \\
\hline BMI1 & Hs00995522_g1 & 153 \\
\hline LRIG1 & $\underline{\text { Hs00394267_m1 }}$ & 96 \\
\hline HOPX & $\underline{\text { Hs04188695_m1 }}$ & 56 \\
\hline TERT & $\underline{\text { Hs00972650_m1 }}$ & 57 \\
\hline WIP1 & $\underline{\text { Hs01013292_m1 }}$ & 65 \\
\hline KRT19 & $\underline{\text { Hs00761767_s1 }}$ & 116 \\
\hline KI67 & $\underline{\text { Hs00606991_m1 }}$ & 137 \\
\hline HES1 & Hs00172878_m1 & 78 \\
\hline DLL1 & $\underline{\text { Hs00194509 m1 }}$ & 74 \\
\hline CHGA & $\underline{\text { Hs00900370_m1 }}$ & 67 \\
\hline MMP7 & $\underline{\text { Hs01042796_m1 }}$ & 64 \\
\hline ATOH1 & $\underline{\text { Hs00944192_s1 }}$ & 83 \\
\hline TFF3 & $\underline{\text { Hs00902278_m1 }}$ & 64 \\
\hline CCK & $\underline{\text { Hs00174937 m1 }}$ & 69 \\
\hline LYZ & $\underline{\text { Hs00426232_m1 }}$ & 67 \\
\hline
\end{tabular}


Data Reporting. The experiments were not randomized. The investigators were not blinded during experimental setup or evaluation of experimental outcomes. No statistical methods were used to determine sample size.

Tissue processing. De-identified tissue biopsies were collected from unaffected intestinal regions of children and adult patients undergoing endoscopy for gastrointestinal complaints. Informed consent from adult donors or from a pediatric donors' guardian along with developmentally appropriate assent donor were obtained at Boston Children's Hospital. All methods were carried out in accordance with the Institutional Review Board of Boston Children's Hospital (Protocol number IRB-P00000529). Tissue processing and initial human and mouse organoid culture were performed at the Harvard Digestive Disease Center, following published protocols ${ }^{1,2}$. Organoids were grown in growth factor reduced (GFR) Matrigel droplets overlaid with expansion medium

14 (EM) made with L-WRN conditioned medium (50\% vol/vol, ATCC, CRL3276) and supplemented with Y-27632 (10 $\mu \mathrm{M}$, TOCRIS $)^{1,2}$. Mouse colon organoids were cultured as previously reported ${ }^{3}$.

16 Endometrial tissue collection was approved by the Partners Healthcare Institutional Review Board (Protocol number IRB-P001994). De-identified uterine tissue or pipelle biopsies were collected from consented adult donors (ages 18-45). Endometrial organoids were established using published protocols and cultured in endometrial organoid medium (EnOM) ${ }^{5,6}$.

20 Cell culture. Organoids were passaged every four days for human intestinal organoids, eight days

21 for human endometrial organoids and three days for mouse organoids. For passaging, organoids were incubated in Cell Recovery Solution (CRS, Gibco) for 1h at 4C, followed by trypsin treatment

23 to generate single cells. The cell suspension was inspected under the microscope to ensure the 24 presence of dispersed single cells and if needed, filtered through a $30 \mu \mathrm{m}$ cell strainer to remove 25 cell clumps. Single cells were counted using a hemocytometer and seeded in GFR Matrigel (25 $26 \mu \mathrm{L}$ droplet) at a density of 1,000 cells $/ \mu \mathrm{L}$ for human and 500 cells/ $\mu \mathrm{L}$ for mouse organoids. After 27 Matrigel polymerization, $600 \mu \mathrm{L}$ of EM or EnOM was layered on top. Media was changed every 28 two days.

29 Organoid differentiation. Organoids in both Matrigel and the synthetic matrix were cultured in 30 EM for six days (human) or four days (mouse) before switching to differentiation medium. Human 31 and mouse organoids in Matrigel were differentiated as previously reported ${ }^{1,2}$. Intestinal organoids 32 in the synthetic matrix were differentiated using L-WRN conditioned medium (25\% L-WRN) 
1 diluted in Adv DMEM/F12, HEPES (1 $\mu \mathrm{M}$, Gibco), Pen/Strep (100 U/mL, $100 \mathrm{mg} / \mathrm{mL}$,

2 Invitrogen), N2 supplement (1X, Gibco), B27 supplement (1X, Gibco), human [Leu15]-gastrin I

3 (10 nM, BACHEM), N-acetyl cysteine (1 mM, SIGMA) and Y-27632 (5 $\mu$ M, TOCRIS). Mouse

4 organoids in the synthetic ECM were differentiated as in Matrigel.

5 PEG macromers and peptides. 8-arm PEG macromers with vinyl sulfone (VS) terminal groups

6 (40kDaPEG-VS and 20kDaPEG-VS) were obtained from JenKem Technology (Beijing). All

7 peptides were custom synthesized and purified by Boston Open Labs (Cambridge, MA), GenScript

8 (Piscataway, NJ), or CPC Scientific (Sunnyvale, CA). Peptides sequences and their relevant

9 features are included in the Supplementary Table 1. "XL-VP," a crosslinker containing a matrix

10 metalloproteinase (MMP)-sensitive substrate, "XL-IA," a crosslinker containing a MMP-sensitive

11 substrate and a Sortase-sensitive recognition site (LPRTG), "XL," a crosslinker containing only

12 the Sortase-sensitive site, "RGDS,'” a fibronectin (FN)-derived peptide containing the canonical

13 RGD motif from the 10th FN type III domain, "PHSRN-K-RGD,' a FN-derived peptide

14 containing the RGD motif and the PHSRN synergy site from the 9th FN type III repeat in a

15 branched configuration; “GFOGER,'” a collagen I-derived peptide presented as a triple helix ${ }^{8,9}$,

16 "GFOGDR," a collagen I-derived peptide with $\mathbf{E} \rightarrow \mathbf{D}$ point mutation that reduces integrin

17 binding ${ }^{10}$, “G11RGDS," an extended ligand of RGDS with eleven Gly spacers, "CMPRGDS,” an

18 extended and clustered RGDS ligand using a triple helical collagen mimetic peptide ${ }^{11}$, "FN-

19 binder,' a peptide with affinity for FN, and 'BM-binder,' a peptide with affinity for collagen

20 IV (C-IV) and laminin (LMN) ${ }^{12}$. All peptides were reconstituted in acidic (pH 5.2) Milli-Q water

21 (Millipore). The concentration of free thiols in all peptides was determined using Ellman's reagent

22 (Sigma).

23 Synthesis of hydrogel precursors. All synthetic matrices were assembled at $5 \%$ PEG (w/v) using

24 8-arm 40kDaPEG-VS or 8-arm 20kDaPEG-VS. The mixed 40/20kDaPEG synthetic matrix was

25 made mixing 1:1 (v/v, 2.5\% 8-arm 40kDaPEG-VS and 2.5\% 8-arm 20kDaPEG-VS). All matrices

26 were assembled stepwise via Michael-type addition reaction. First, the PEG-VS macromer was

27 diluted with 10X PBS-HEPES solution (1 mM, pH 7.8); second, the matrix-binder peptides were

28 added to the PEG reaction mixture and incubated for 30 minutes at RT; third, the integrin binder

29 peptides (RGDS, PHSRN-K-RGD, GFOGER, G11RGDS, CMPRGDS or GFOGDR) were

30 added to the reaction mixture and allowed to react for an additional $30 \mathrm{~min}$ at RT. This sequential

31 reaction created a PEG-functionalized mixture "fPEG-VS", that was used to resuspend the cells 
1 prior to matrix gelation (see cell encapsulation below). The nominal concentration of the matrix-

2 binders in all matrices was $0.25 \mathrm{mM}$ each, whereas the integrin binder peptides were at $1.5 \mathrm{mM}$

3 (unless otherwise noted in the figure legends). For most experiments the fPEG-VS solution was

4 crosslinked at 50\% crosslinking density (unless otherwise noted in the figure legends).

5 Rheological characterization. After adding the XL-IA crosslinker (50\%), $20 \mu \mathrm{L}$ of each matrix

6 mixture was loaded into a $1 \mathrm{~mL}$ syringe that had the tip cut off at the $0.1 \mathrm{~mL}$ mark. The matrix

7 was allowed to gel at $37^{\circ} \mathrm{C}$ for $20 \mathrm{~min}$ and then moved to a 24 well plate that contained $400 \mu \mathrm{L}$ of

8 1X PBS. The plate was incubated for 24 hours in a humidified incubator at $37{ }^{\circ} \mathrm{C}, 95 \%$ air, and

$95 \% \mathrm{CO}_{2}$, to allow for equilibrium swelling to occur prior to rheological characterization. This

10 procedure created matrices discs of 1-1.4 $\mathrm{mm}$ in thickness. The discs were sandwiched between

11 an $8 \mathrm{~mm}$ sandblasted parallel plate and sandblasted base. The shear modulus was determined by

12 performing small-strain oscillatory shear measurements on an Anton Parr MCR 302 instrument.

13 The mechanical response was recorded by performing frequency sweep measurements $(0.1-10$

$14 \mathrm{~Hz})$ in a constant strain mode $(0.05)$, at $37^{\circ} \mathrm{C}$. The shear modulus $\left(\mathrm{G}^{\prime}\right)$ is reported as a measure of

15 matrix mechanical properties.

16 Equilibrium swelling. The matrices were prepared at 50\% crosslinking density (unless otherwise

17 noted in the figure legends) as described above. After adding the XL-VP crosslinker, three 30- $\mu \mathrm{L}$

18 droplets were loaded onto three 18-mm circular glass micro-coverslips.

19 The mass of the coverslip (mCs) were determined prior to the addition of the gel mixture to

20 calculate the percentage of swelling (see below). The gel mixture/coverslips were placed inside of

21 a 12-well plate and allowed to gel for $20 \mathrm{~min}$ in a humidified incubator at $37{ }^{\circ} \mathrm{C}, 95 \%$ air, and $5 \%$

$22 \mathrm{CO}_{2}$. At the end of the gelation, each coverslip was weighed again and the mass recorded as "pre-

23 swelled matrix mass (mpSM)". One mL of $1 \mathrm{X}$ PBS was loaded onto each matrix droplet and

24 returned to the incubator for 24 hours to allow the matrix to reach equilibrium swelling. After 24

25 hours, the PBS was removed, and the matrices were washed twice with $1 \mathrm{~mL}$ of $\mathrm{dH}_{2} \mathrm{O}$. The water

26 was removed completely before the matrix/coverslips were weighed again. This was recorded as

27 "swelled matrix mass (mSM)". Finally, the matrix/coverslips were placed in a $60{ }^{\circ} \mathrm{C}$ oven

28 overnight to determine the mass of the dry matrix. To calculate the percentage of swelling we sued

29 the following formula $((\mathrm{mpSM}-\mathrm{mCs}) /(\mathrm{mSM}-\mathrm{mCs}))^{*} 100$. The pore size $(\xi)$ was calculated

30 according to Flory-Rehner equations and derived formulas described previously ${ }^{13}$. In experiments

31 to determine the effect of the crosslinking density on swelling and pore size, the synthetic matrices 
1 were made with $35,40,45,50,55$, and $60 \%$ XL-VP crosslinker density. The percentage of swelling

2 and pore size were calculated as above.

3 Cell encapsulation. Four-day old organoids grown in Matrigel were collected and processed as

4 above to generate single cells. The cell suspension was inspected under the microscope to ensure

5 the presence of dispersed single cells and if needed, filtered through a $30 \mu \mathrm{m}$ cell strainer to remove

6 cell clumps. Single cells were counted using a hemocytometer then resuspended in the matrix

7 precursor solutions (fPEG-VS) prior to the addition of the crosslinker and Y-27632 (10 $\mu \mathrm{M})$. In

8 parallel, single cells were resuspended in Matrigel that served as experimental control during

9 matrix evaluation. In both cases, cells were encapsulated at a density of 500 cells/ $\mu \mathrm{L}$ of matrix.

10 Three $\mu \mathrm{L}$ (1,500 cells) of the matrices were loaded into a Nunc MicroWell 96-well optical-Bottom

11 plate and allowed to polymerase for $20 \mathrm{~min}$ in a humidified incubator at $37{ }^{\circ} \mathrm{C}, 95 \%$ air, and $5 \%$

$12 \mathrm{CO}_{2}$. After gelation, $100 \mu \mathrm{L}$ of EM or EnOM was loaded into each well. Media was changed every

13 two days. Eight-day old endometrial organoids were used to generate single cells. The

14 encapsulation process in Matrigel or the synthetic ECM, for endometrial organoids, was performed

15 similar to intestinal organoids.

16 Intestinal and endometrial enteroid/organoid diameter. The diameter of six-day old enteroids

17 and eight-day old endometrial organoids were determined using a deep learning based algorithm

18 as described previously ${ }^{14}$. $4 \mathrm{X}$ brightfield (BF) images were captured using an EVOS M500

19 microscope (Invitrogen).

20 Enteroid formation efficiency. Single cells were encapsulated as above and cultured in EM for

21 four days (Matrigel) or six days (synthetic matrix) before imaging at 10X magnification using an

22 EVOS M500 microscope. Sixty BF images, spanning the entire thickness of the matrix, were

23 collected from the center of the droplet. This is approximately $1 / 3$ of the total droplet volume. The

24 image z-stacks were processed in Fiji using the time lapse Gaussian-based stacker focuser plugin

25 to create a single image with all enteroids in focus. The number of enteroids with a clear lumen

26 and the number of single cells were manually counted using the cell counter feature in Fiji ${ }^{15}$. We

27 also observed and counted few cell clumps that did not show a clear lumen but were bigger than

28 single cells. Enteroid formation efficiency was calculated as the percentage of enteroids with a

29 clear lumen relative to the total number of enteroids, single cells, and cell clumps, counted in each

30 droplet. 
1 Live/dead imaging. Single cells were encapsulated as before for six days (intestinal) or eight days

2 (endometrial) before the addition of Calcein AM (2 mM) and Ethidium homodimer-1 (2 mM) for

320 minutes. Images were captured using either a ZEISS confocal Laser Scanning Microscope

4 (LSM 880) equipped with temperature $\left(37^{\circ} \mathrm{C}\right)$, humidity, and $\mathrm{CO}_{2}(5 \%)$ controls orr an EVOS

5 M500 (Invitrogen) microscope (no incubation). A $1.6 \mathrm{~mm}$ by $1.6 \mathrm{~mm}$ area and $\sim 800 \mu \mathrm{m}$ thick

6 section of either Matrigel or the synthetic ECM were imaged with the confocal. With the EVOS,

7 we captured the center of the droplet, which is $\sim 1 / 3$ of the total matrix area. The final images were

8 processed using the ZEN blue ZEISS companion software or Fiji ${ }^{15}$. For time-course live/dead

9 imaging analysis, 60 z-stacks images were taken the day of seeding (day 0) then every two days

10 for up to ten days, using the EVOS M500 microscope and processed as above.

11 Continues live imaging. Single cells were encapsulated and plated on 96-well optical plates as

12 described before, then cultured in OEM. Bright field images were captured using a ZEISS confocal

13 Laser Scanning Microscope (LSM 880) equipped with a wide-field camera and temperature (37

$\left.14{ }^{\circ} \mathrm{C}\right)$, humidity, and $\mathrm{CO}_{2}(5 \%)$ controls. A single plane of four-day old enteroids were imaged every

155 minutes for 48 hours. The final videos were prepared using Fiji using frame interpolation to

16 smooth the video ${ }^{15}$. To capture individual cells forming enteroids, the entire thickness of the

17 matrices was imaged the day after encapsulation and then the everyday for up to six days. The

18 stack of images was processed in Fiji as described in enteroid formation efficiency ${ }^{15}$.

19 Cell proliferation. To determine if cells from enteroids in the synthetic ECM retain their

20 proliferative capacity, we collected six-day old enteroids from within the synthetic ECM using

21 SrtA treatment ${ }^{16}$, then digested them with trypsin to generate a single cell suspension. Cells (500

22 cells $/ \mu 1,5 \mu l$ droplets, 48 droplets total) were embedded in Matrigel or the synthetic ECM and

23 cultured in EM for six days. After six days, the matrix droplets were pooled and processed as

24 follows; enteroids in Matrigel were released using CRS whereas enteroids in the synthetic ECM

25 were released using SrtA treatment. Enteroids in both conditions were then digested with trypsin

26 to get the total number of cells recovered from the pooled enteroids, from each matrix condition.

27 The fold increase in cell number was calculated as the ratio of total number of cells obtained from

28 enteroids after six days of culture divided by the total number of cells used at the beginning of the

29 experiment. The cells recovered from enteroids in Matrigel were discarded. The cells recovered

30 from enteroids in the synthetic ECM were used to set up a new experiment (first passage, 500

31 cells $/ \mu 1,5 \mu$ d droplets, 48 droplets total) in new synthetic ECM or Matrigel. At the end of six days, 
1 the fold increase in cell number was determined as before. This process was repeated three consecutive passages using two human duodenal donors. In parallel experiments, using the same pool of cells, we measured the enteroid diameter of the three consecutive passagesas previously 4 described $^{14}$.

5 Quantitative real time PCR (qPCR). Four-day old enteroids grown in Matrigel were used to generate a single-cell suspension as described above. Single cells (500 cells $/ \mu \mathrm{L})$ were encapsulated in Matrigel or the synthetic ECM, then cultured in EM. After six days, intact enteroids were released from Matrigel and the synthetic ECM, as described before. Intact enteroids were pelleted, resuspended in TRIzol reagent (ThermoFisher Scientific, 15596026), and then stored at $-80{ }^{\circ} \mathrm{C}$ until processing. From the initial cell suspension, we reserved 500,000 cells at $-80{ }^{\circ} \mathrm{C}$ in TRIzol.

11 This single-cell population was used to determine the gene expression of the "initial cell 12 population". RNA was extracted from enteroids (or cells) using the Directzol RNA Mini-Prep kit 13 (Zymo Research) per the manufacturer's protocols with the inclusion of an on-column DNase step 14 using the PureLink DNase Set (Thermofisher Scientific, 12185010). cDNA was synthesized from $15 \sim 1 \mu \mathrm{g}$ of total RNA using the High-Capacity RNA-to-cDNA Kit (Thermofisher Scientific, 4387406) per manufacturer's protocols. TaqMan Fast Advanced Master Mix (Thermofisher

17 Scientific, 4444557) was used in congruence with the cell-specific probes for qPCR described in 18 the supplementary Table 2. Gene expression was determined using the StepOnePlus real-time PCR 19 system (Applied Biosystems) and calculated using the $\Delta \Delta \mathrm{Ct}$ method in GraphPad Prism. Gene 20 expression was first normalized using the housekeeping GAPDH gene in each sample, then the 21 relative fold change in gene expression, in Matrigel or the synthetic ECM, was calculated against 22 the gene expression of the "initial cell population" that was set to 1 . The experiment was repeated 23 three times with two duodenal donors.

24 Histological processing and immunostaining. Mouse intestinal organoids were fixed in while still in the gel (3D), then paraffin-embedded and sectioned in the Histology Center at the Koch 26 Institute at MIT. 5-micron tissue sections were hematoxylin and eosin stained using standard 27 procedures.

28 Immunostaining and EdU labeling. Organoids and enteroids were processed in two formats: in $293 \mathrm{D}$ (embedded within the synthetic ECM or Matrigel) and in suspension (free floating) after being 30 released from the matrices. Six-day old enteroids in 3D were treated with EdU (5-ethynyl-2'31 deoxyuridine, $20 \mu \mathrm{M}$ ) for $3 \mathrm{hr}$ (Alexa Flour 488 Click-it EDU, Thermo Fisher) prior fixation 
1 overnight with formalin $(10 \%, \mathrm{VWR})$ at $4{ }^{\circ} \mathrm{C}$. Eight-day old endometrial organoids in 3D were treated with EdU for $6 \mathrm{hr}$ prior to overnight fixation. Eight-day old colon organoids were treated with EdU for $6 \mathrm{hr}$, then released from the matrices and fixed as free-floating organoids for $30 \mathrm{~min}$ at RT. After fixation, organoids and enteroids in 3D were permeabilized with $0.1 \%$ triton X-100 in PBS overnight followed by blocking (4\% BSA/0.5\% Tween 20 in 1 XPBS or 4\% Donkey serum $/ 0.5 \%$ Tween 20 in $1 \mathrm{X}$ PBS) overnight at $4^{\circ} \mathrm{C}$ with $200 \mathrm{rpm}$ shaking. Free-floating organoids were permeabilized for $1 \mathrm{~h}$ at RT incubated in a tube rotator set at $30 \mathrm{rpm}$, followed by $3 \mathrm{hr}$ of blocking. To identify proliferative cells, the Alexa Flour 488 was click-reacted according to the manufacturer instruction. Immunostaining for cell-specific markers was done using the following primary antibodies, diluted in blocking solution; goat anti-E-cadherin (R\&D, AF748, 1:200), goat anti-hDPPIV/CD26 (Invitrogen, AF1180, 1:400), rabbit anti-Col IV (Abcam, Ab6586, 1:200), rabbit anti-Lysozyme (Dako, A0099, 1:200), rabbit anti-LMN (Abcam, Ab11575, 1:200), rabbit 13 anti-Ki67, (Abcam, Ab15580, 1:200 or ab16667, 1:100), rabbit anti-NHE3/SCL9A3 (Novus, 14 NBP-82574), mouse anti-Villin (Santa Cruz, SC-58897, 1:50), mouse anti-CD44-v6 (Abcam, Ab78960, 1:200), mouse anti-Muc2 (Santa Cruz, SC515032 1:200), mouse anti-EpCAM (Abcam, ab7504 1:200). Samples in 3D were incubated for two days in primary antibodies at $4^{\circ} \mathrm{C}$ and 200

17 rpm shaking. Free-floating samples were incubated overnight in primary antibodies at $4^{\circ} \mathrm{C}$ in the tube rotator set at $30 \mathrm{rpm}$. The secondary antibodies and dilutions used were; donkey anti-goat Alexa F1 568 (ThermoFisher, A11057, 1:200), donkey anti-rabbit Alexa F1 568 (ThermoFisher, A10042, 1:200), and donkey anti-mouse Alexa F1 568 (ThermoFisher, A10037, 1:200). Nuclear staining was done using DAPI (1 mg/mL, 1:2,000). F-actin staining was done using either Alexa F1 488 phalloidin (ThermoFisher, A12379, 1:200) or Alexa F1 568 (ThermoFisher, A12380, 1:200). DAPI and f-actin staining was done in combination with the secondary antibody. Samples in 3D were incubated in secondary antibodies for two days at ${ }^{\circ} \mathrm{C}$ and $200 \mathrm{rpm}$ shaking. At the end of the incubation, samples in 3D were washed five times (10 minutes each, $200 \mathrm{rpm}$ at RT) prior to the addition of CytoVista reagent (Thermofisher) to match the refraction index and allow imaging in 3D. Free-floating samples were incubated overnight in secondary antibodies at $4{ }^{\circ} \mathrm{C}$ in the tube rotator set at $30 \mathrm{rpm}$, followed by five washes (10 min each, at RT and $30 \mathrm{rpm})$. The 29 organoids were gently pelleted and resuspended in prolong gold antifade reagent (ThermoFisher, P36935), then transferred onto a glass slide modified with a $20 \mathrm{~mm}$ secure seal spacer $(0.12 \mathrm{~mm}$

31 deep, ThermoFisher, S24736). 5-micron tissue sections were incubated in mouse anti-Villin (Santa 
1 Cruz, SC-58897, 1:50) overnight, followed by donkey anti-mouse Alexa Fl 568 (ThermoFisher,

2 A10037, 1:200) and DAPI staining. Images were captured using a ZEISS confocal Laser Scanning

3 Microscope (LSM 880).

4 Statistical analysis and sample information. Statistical significance between experimental 5 treatments were determiend as follows: Enteroid diameters were analyzed using one-way ANOVA and Kruskal-Wallis multiple comparison of the mean ranks. Storage modulus data analyzed using one-way ANOVA and Sidak's multiple comparison whereas swelling and pore size data was analyzed using Kruskal-Wallis multiple comparison of the mean ranks. Enteroid formation was analyzed using either one-way ANOVA and Holm-Sidak's multiple comparison of the mean or two tailed Mann-Whitney tests when comparing two groups. qPCR data were analyzed using

11 multiple $\mathrm{t}$ tests and the Holm-Sidak method. All statistical analyses were performed in the 12 GraphPad Prism 8.0 software.

Data availability. Source Data for Figs. 1-6 are provided with the paper. All additional relevant data are available upon request from the corresponding author.

Acknowledgements. We thank the Harvard Digestive Disease Center for establishing the original intestinal cultures from biopsies. Professor Thaddeus Stappenbeck from Washington University School of Medicine in St. Louis for providing the mouse colon organoids and the L-WRN cell line to produce the conditioned medium. We thank the Koch Institute histology and microscopy centers at MIT for helping establish protocols for organoid processing. We thank Alexander Brown (MIT) for helpful discussions of the manuscript, Dr. Katherine Bezold Lamm (MIT) for help collect some samples for qPCR analysis and for her helpful comments on the manuscript and Derrick Marsall (MIT) for help in the rheological characterization. We thank the MIT UROP office for financial support to A. L. and G.C. This work was supported by funding from NIH Research Grant No. R01EB021908 and by financial support from DARPA and Boehringer Ingelheim through the SHINE program.

28 Author Contributions. V.H.G. and L.G.G conceived the study, designed experiments and wrote 29 the manuscript. V.H.G. cultured organoids and performed the experiments with the help of A. L,

30 G. C. and M.G. All were involved in data collection whereas V.H.G was involved in processing 31 and analyzeing all data. A.L. performed matrix characterization. T.K. conceived and developed 32 the algorithm to measure the enteroids, help processing data and edited the manuscript. J.G.N. 
1 collected, processed and established human endometrial organoids and performed immunostaining

2 of endometrial organoids. R.C. edited the manuscript. D.B. supervised tissue collection to establish

3 initial organoid culture, edited the manuscript.

Supplementary Table 1| Peptide sequences and relevant features

\begin{tabular}{|c|c|c|}
\hline Name & Sequence & Relevant Feature \\
\hline XL-VP & (Ac)-GCRD-VPMSMRGG-DRCG-(Am) & $\begin{array}{l}\text { a dithiol peptide containing a matrix } \\
\text { metalloproteinase (MMP)-sensitive substrate }\end{array}$ \\
\hline XL-IA & (Ac)-GCRD-LPRTG-GPQGIAGQ-DRCG-(Am) & $\begin{array}{l}\text { a dithiol peptide containing a MMP-sensitive } \\
\text { substrate and a Sortase-sensitive recognition } \\
\text { site (LPRTG) }\end{array}$ \\
\hline XL & (Ac)-GCRD-LPRTG-DRCG-(Am) & $\begin{array}{l}\text { a dithiol peptide containing only the Sortase- } \\
\text { sensitive site (LPRTG) }\end{array}$ \\
\hline RGDS & (Ac)-GCRE-RGDSP-(Am) & $\begin{array}{l}\text { a fibronectin (FN)-derived peptide containing } \\
\text { the canonical RGD motif from the } 10 \text { th FN } \\
\text { type III domain }\end{array}$ \\
\hline $\begin{array}{l}\text { PHSRN-K- } \\
\text { RGD }\end{array}$ & (Ac)-PHSRNGGGK-(GGG-ERCG-(Am))-GGRGDSPY-(Am) & $\begin{array}{l}\text { a FN-derived peptide containing the RGD } \\
\text { motif and the PHSRN synergy site from the } \\
\text { 9th FN type III repeat in a branched } \\
\text { configuration, }\end{array}$ \\
\hline GFOGER & “"(Ac)-GGYGGGPG(GPP) $)_{5}$ GFOGER(GPP) $)_{5}$ GPC-(Am) $)^{8,9}$ & $\begin{array}{l}\text { a collagen I-derived peptide presented as a } \\
\text { triple helix, }\end{array}$ \\
\hline GFOGDR & (Ac)- GGYGGGPG(GPP) ${ }_{5}$ GFOGDR(GPP) ${ }_{5}$ GPC-(Am) ${ }^{10}$ & $\begin{array}{l}\text { a collagen I-derived peptide with a point } \\
\text { mutation } \mathbf{E} \rightarrow \mathbf{D} \text { that reduces integrin binding }\end{array}$ \\
\hline G11RGDS & (Ac)-GCRE-GGGGGGGGGGGRGDSP-(Am), & $\begin{array}{l}\text { an extended ligand of RGDS with eleven Gly } \\
\text { spacers }\end{array}$ \\
\hline CMPRGDS & $(\mathrm{Ac}) \mathrm{CGG}(\mathrm{POG}){ }_{9} \mathrm{RGDSP}(\mathrm{Am})^{11}$ & $\begin{array}{l}\text { an extended and clustered RGDS ligand using } \\
\text { a triple helical collagen mimetic peptide }\end{array}$ \\
\hline FN-binder & (Am)-GCRE-TLQPVYEYMVGV-(Ac) & a peptide with affinity for $\mathrm{FN}$ \\
\hline BM-binder & Ac-GCRE-ISAFLGIPFAEPPMGPRRFLPPEPKKP-(Am) ${ }^{12}$ & $\begin{array}{l}\text { a peptide with affinity for collagen IV (C-IV) } \\
\text { and laminin (LMN) }\end{array}$ \\
\hline
\end{tabular}

1. Kasendra, M. et al. Development of a primary human Small Intestine-on-a-Chip using biopsy-derived organoids. Sci. Rep. 8, 2871 (2018).

2. Miyoshi, H. \& Stappenbeck, T. S. In vitro expansion and genetic modification of gastrointestinal stem cells in spheroid culture. Nat. Protoc. 8, 2471-82 (2013).

3. Miyoshi, H. et al. Prostaglandin E2 promotes intestinal repair through an adaptive cellular response of the epithelium. EMBO J. 36, 5-24 (2017).

4. Richmond, C. A. et al. JAK/STAT-1 Signaling Is Required for Reserve Intestinal Stem Cell Activation during Intestinal Regeneration Following Acute Inflammation. Stem Cell Reports 10, 17-26 (2018).

5. Turco, M. Y. et al. Long-term, hormone-responsive organoid cultures of human endometrium in a chemically defined medium. Nat. Cell Biol. 19, 568-577 (2017).

6. Boretto, M. et al. Patient-derived organoids from endometrial disease capture clinical heterogeneity and are amenable to drug screening. Nat. Cell Biol. 21, 1041-1051 (2019).

7. Yan, K. S. et al. Non-equivalence of Wnt and R-spondin ligands during Lgr5(+) intestinal stem-cell selfrenewal. Nature 545, 238-242 (2017).

8. Wojtowicz, A. M. et al. Coating of biomaterial scaffolds with the collagen-mimetic peptide GFOGER for bone defect repair. Biomaterials 31, 2574-2582 (2010).

9. Carafoli, F., Hussain, S.-A. \& Hohenester, E. Crystal Structures of the Network-Forming Short-Arm Tips of the Laminin b1 and c1 Chains. (2012). doi:10.1371/journal.pone.0042473

10. Knight, C. G. et al. The collagen-binding a-domains of integrins $\alpha 1 / \beta 1$ and $\alpha 2 / \beta 1$ recognize the same specific amino acid sequence, GFOGER, in native (triple- helical) collagens. J. Biol. Chem. 275, 35-40 
1 (2000).

11. Hernandez-Gordillo, V. \& Chmielewski, J. Mimicking the extracellular matrix with functionalized, metalassembled collagen peptide scaffolds. Biomaterials 35, 7363-7373 (2014).

12. Johnson, G. \& Moore, S. W. Identification of a structural site on acetylcholinesterase that promotes neurite outgrowth and binds laminin-1 and collagen IV. Biochem. Biophys. Res. Commun. 319, 448-455 (2004).

13. Zustiak, S. P. \& Leach, J. B. Hydrolytically degradable poly(ethylene glycol) hydrogel scaffolds with tunable degradation and mechanical properties. Biomacromolecules 11, 1348-1357 (2010).

14. Kassis, T., Hernandez-Gordillo, V., Langer, R. \& Griffith, L. G. OrgaQuant: Human Intestinal Organoid

15. Schindelin, J. et al. Fiji: An open-source platform for biological-image analysis. Nature Methods 9, 676-682 (2012).

16. Valdez, J. et al. On-demand dissolution of modular, synthetic extracellular matrix reveals local epithelialstromal communication networks. Biomaterials 130, 90-103 (2017). 